\title{
The Use of Biomarkers in Clinical Management Guidelines: A Critical Appraisal
}

\author{
María Asunción Esteve-Pastor ${ }^{1}$ () Vanessa Roldán ${ }^{2}$ \\ Inmaculada Ramírez-Macías ${ }^{1}$ Gregory Y. H. Lip ${ }^{3,4}$ \\ ${ }^{1}$ Department of Cardiology, Hospital Clínico Universitario Virgen de \\ la Arrixaca, Instituto Murciano de Investigación Biosanitaria \\ (IMIB-Arrixaca), CIBERCV, University of Murcia, Murcia, Spain \\ 2 Department of Hematology and Clinical Oncology, Hospital \\ Universitario Morales Meseguer, Instituto Murciano de Investigación \\ Biosanitaria (IMIB-Arrixaca), University of Murcia, Murcia, Spain \\ ${ }^{3}$ Liverpool Centre for Cardiovascular Science, Liverpool Heart \& Chest \\ Hospital, University of Liverpool, Liverpool, United Kingdom \\ ${ }^{4}$ Department of Clinical Medicine, Aalborg Thrombosis Research \\ Unit, Aalborg University, Aalborg, Denmark
}

José Miguel Rivera-Caravaca1(1)

Francisco Marín ${ }^{1}$

\begin{abstract}
Address for correspondence Francisco Marín, MD, PhD, Department of Cardiology, Hospital Clínico Universitario Virgen de la Arrixaca, IMIB-Arrixaca, Ctra. Madrid-Cartagena s/n 30120, Murcia, Spain (e-mail: fcomarino@hotmail.com).
\end{abstract}

Thromb Haemost 2019;119:1901-1919.

\begin{abstract}
Keywords

- vascular cell markers

- cardiology

- management of disease

In cardiovascular disease (CVD), biomarkers (i.e., "biological markers") could have multiple roles in understanding the complexity of cardiovascular (CV) pathophysiology and to offer an integrated approach to management. Biomarkers could help in daily practice as a diagnostic tool, to monitor therapy response, to assess prognosis and as early marker of CV damage, or to stratify risk. In recent years, the role of biomarkers in CVD is even more relevant and some have recently been included in clinical management guideline recommendations. The aim of this review is to discuss the recommendations in clinical guidelines of various biomarkers and to review their usefulness in daily clinical practice. Ultimately, a balance is needed between simplicity and practicality for clinical decisionmaking. Most biomarkers (whether blood, urine, or imaging-based) will improve on clinical risk stratification, but awaiting biomarker results may lead to delays in the initiation of therapy, for example, anticoagulation for stroke prevention in atrial fibrillation. Many biomarkers are nonspecific, being predictive of many CV and non-CV outcomes, so would be better as "rule-out" rather than "rule-in" assessments. Derivation of some biomarkers have also been made in highly selected clinical trial cohorts, where measurement is made at baseline but outcomes determined many years later; given the dynamic nature of risk in the "real world" where patients get older and develop incident risk factors, this may give a false impression of the risk profile. Finally, some laboratory biomarkers have a diurnal variation and inter-/intravariability (and lower limits of detection) in assays, which may be expensive, are added considerations.
\end{abstract}

\section{Introduction}

In cardiovascular disease (CVD), biomarkers (i.e., "biological markers") could have multiple roles in understanding the complexity of cardiovascular (CV) pathophysiology and to

received

May 10, 2019

accepted after revision

July 15, 2019

offer an integrated approach to management. The Food and Drug Administration (FDA) defines a biomarker as any measurable indicator that is potentially useful throughout the whole spectrum of the disease process; research and development of new therapies; diagnosis, prognosis, and monitoring

(c) 2019 Georg Thieme Verlag KG Stuttgart - New York
DOI https://doi.org/

10.1055/s-0039-1696955. ISSN 0340-6245. 
progression of a disease; or response to treatment. ${ }^{1}$ Biomarkers could help in daily practice as a diagnostic tool, to monitor therapy response, to assess prognosis and as early marker of $\mathrm{CV}$ damage, or to stratify risk. ${ }^{2}$ However, the implementation of routine strategies in a cost-effective manner is restricted by the limited predictive value of current risk assessment models using biomarkers. ${ }^{3}$

In recent years, the role of biomarkers in CVD is even more relevant and some have recently been included in clinical management guideline recommendations. The aim of this review is to discuss the recommendations in clinical guidelines of various biomarkers and to review their usefulness in daily clinical practice.

\section{Biomarkers in Heart Failure}

Heart failure (HF) is associated with many different cardiac and extracardiac pathophysiological mechanisms leading to a complex clinical syndrome with multiple phenotypes. The diagnosis of $\mathrm{HF}$ can be difficult because the clinical presentation does not necessarily imply structural cardiac involvement. It may be important to develop specific and immediate tests to perform a rapid "rule-out" of HF in the emergency department.

Recent studies have highlighted the role of different biomarkers to assess patients with HF, which have focused on different pathways: myocardial stress, neurohormonal activation, inflammatory state, or remodeling. Hence, these biomarkers are not only related to the diagnosis in the acute stress phase of $\mathrm{HF}$ but also to the chronic state ${ }^{4}$ which could help in daily clinical practice for monitoring the response of a new therapy or to stratify the prognosis related to future admissions. Moreover, these markers can identify early heart transplant rejection or cardiotoxicity with antineoplastic agents. ${ }^{5}$

The most important biomarkers in HF patients are the natriuretic peptides (NPs) and troponin levels. Plasma levels of the NPs (B-type NP [BNP] and N-terminal fraction of BNP [NT-proBNP]) are the result of end-diastolic stress due to an increase in volume or pressure. BNP is a neurohormone synthesized by myocytes in response to increased cardiac wall tension. ${ }^{6}$ In the setting of volume expansion or pressure overload, the resulting wall stress initiates the synthesis of pre-proBNP in the ventricular myocardium, mainly in the left ventricular (LV) myocardium. ${ }^{7}$ After that, the peptide is cleaved in the active BNP and in the inactive aminoterminal NT-proBNP form. ${ }^{6,8}$ However, the use of BNP to guide treatment in $\mathrm{HF}$ patients treated with sacubitril/valsartan is controversial. NPs are substrates of neprilysin; hence, BNP concentrations rise with neprilysin inhibition. The clinical utility of BNP in sacubitril/valsartantreated patients has been questioned, and NT-proBNP has been recommended. Myhre et $\mathrm{al}^{9}$ showed that despite an initial increase in BNP after initiation of sacubitril/valsartan in approximately $60 \%$ of patients, which may lead to clinical confusion, BNP remained a reliable prognostic marker before and during treatment with sacubitril/valsartan. Cardiac troponins are sensitive and specific markers of myo- cardial injury. Elevations of troponin I and/or troponin T are observed in a many of HF patients without acute coronary syndrome (ACS) due to the stress and damage of myofibrillar proteins.

\section{NT-proBNP for Diagnosis-Making Process and Prognosis in Acute and Chronic Heart Failure (Formal Clinical Guidelines Recommendations in - Table 1)}

2016 European Society of Cardiology Clinical Guidelines of $\mathrm{HF}^{5}$

- The plasma concentration of NPs can be used as an initial diagnostic test, especially in the nonacute setting when echocardiography is not immediately available. Elevated NPs help to establish an initial working diagnosis, identifying those who require further cardiac investigation.

- The upper limit of normality in the nonacute setting for BNP is $35 \mathrm{pg} / \mathrm{mL}$ and for NT-proBNP it is $125 \mathrm{pg} / \mathrm{mL}$. The presence of elevated levels of NPs (BNP $>35 \mathrm{pg} / \mathrm{mL}$ and/or NT-proBNP $>125 \mathrm{pg} / \mathrm{mL}$ ) is used for the algorithm diagnosis of HF in patients with or without reduced ejection fraction. A normal electrocardiogram (ECG) and/or plasma concentrations of BNP $<35 \mathrm{pg} / \mathrm{mL}$ and/or NT-proBNP $<125 \mathrm{pg} / \mathrm{mL}$ make a diagnosis of HF unlikely.

- Cardiac biomarkers (NPs and troponins) can be used to identify patients at higher risk of cardiotoxicity and may be helpful in the monitoring of the use and dosage of cardiotoxic/cytotoxic agents.

2017 ACC/AHA/Task Force on Clinical Practice Guidelines and the Heart Failure Society of America

- During a HF hospitalization, a predischarge NP levels can be useful in establishing a postdischarge prognosis (Class Ila, Level of evidence B).

- Routine determination of BNP or NT-proBNP as preventive tool in patients at risk of $\mathrm{HF}$ is controversial (Class IIa).

Comment on Recommendations in the Clinical Guidelines Clinical guidelines make an important common point about the use of NPs for the diagnosis of acute HF and for the ruling out of $\mathrm{HF}$ in the emergency room. However, the evidence for the latter recommendation is derived from clinical trials and observational registries with moderate sample size.

In a study of 278 patients, Berger et al ${ }^{10}$ demonstrated that the addition of NT-proBNP-guided therapy management in HF patients improved mortality and adverse outcomes rates compared with only $\mathrm{CV}$ risk factors-guided management. The ADHERE (Acute Decompensated Heart Failure National Registry) Registry analyzed 48,629 patients with acute decompensated HF and analyzed BNP quartiles at admission, and found that an elevated admission BNP levels were a significant predictor of in-hospital mortality in acute decompensated HF with either reduced or preserved systolic function, independent of other clinical and laboratory variables. ${ }^{11}$ In the Organized Program to Initiate Lifesaving Treatment in Hospitalized Patients with Heart Failure 
Table 1 Main current clinical guidelines recommendations for biomarkers use in heart failure

\begin{tabular}{|c|c|c|}
\hline & ESC (5) & AHA/ACC(9) \\
\hline \multirow{7}{*}{$\begin{array}{l}\text { Heart } \\
\text { failure }\end{array}$} & $\begin{array}{l}\text { - Measurement of plasma NP level (BNP, NT-proBNP, or } \\
\text { MR-proANP) is recommended in all patients with acute } \\
\text { dyspnea to role-in AHF }\end{array}$ & $\begin{array}{l}\text { - Measuring NP biomarkers (NT-proBNP, or BNP) is } \\
\text { recommended to support a clinical diagnosis of HF }\end{array}$ \\
\hline & Class I, level of evidence $A$ & Class I, level of evidence A \\
\hline & $\begin{array}{l}\text { - In the acute phase of HF, cardiac troponin blood } \\
\text { assessment is recommended to dismiss an ACS }\end{array}$ & $\begin{array}{l}\text { - Measurement of baseline levels of NPs biomarkers } \\
\text { and/or cardiac troponin on admission to the hospital is } \\
\text { useful to establish a prognosis in acutely decompen- } \\
\text { sated HF }\end{array}$ \\
\hline & Class I, level of evidence C & Class I, level of evidence A \\
\hline & $\begin{array}{l}\text { - Multiple other biomarkers, including those reflecting } \\
\text { inflammation, oxidative stress, neurohormonal disarray } \\
\text { and myocardial and matrix remodeling (e.g. ST2, galec- } \\
\text { tin-3, copeptin, adrenomedullin) have been investigated } \\
\text { for their diagnostic and prognostic value in acute HF }\end{array}$ & $\begin{array}{l}\text { - In patients with chronic HF, measurement of other } \\
\text { clinically available tests, such as biomarkers of myo- } \\
\text { cardial injury or fibrosis, may be considered for addi- } \\
\text { tive risk stratification }\end{array}$ \\
\hline & No definite evidence to recommend & Class II b, level of evidence B \\
\hline & $\begin{array}{l}\text { - Renal function should be considered in order to } \\
\text { evaluate the patient's suitability for particular HF } \\
\text { therapies }\end{array}$ & \\
\hline
\end{tabular}

Abbreviations: ACC, American College of Cardiology; ACS, acute coronary syndromes; AHA, American Heart Association; BNP, B-type natriuretic peptide; ESC, European Society of Cardiology; HF, heart failure; NP, natriuretic peptides; NT-proBNP, N-terminal pro-B-type natriuretic peptide; sST2, soluble suppression of tumorigenicity 2.

(OPTIMIZE-HF) trial, ${ }^{12}$ discharge BNP levels were the best 1year predictor of mortality and/or rehospitalization among older patients hospitalized with HF.

NPs have also demonstrated value for predicting new-onset HF and new admissions with this condition. Brouwers et $\mathrm{al}^{13}$ analyzed the role of 13 biomarkers in 8,569 HF-free participants in the Prevention of Vascular and Renal Endstage Disease (PREVEND) study, and the best model for new-onset HF included the combination of NT-proBNP, troponin T, and urinary albumin excretion, especially for those with reduced ejection fraction. The authors suggest that routine biomarker testing should be limited to the use of NPs and troponin T in patients with increased $\mathrm{CV}$ risk.

The cut-off to determine abnormal levels of NPs and diagnose HF is important. Roberts et $\mathrm{al}^{14}$ conducted a systematic review and meta-analyses of two reports that involved 15,263 test results and observed that the lower recommended thresholds of $100 \mathrm{ng} / \mathrm{L}$ for BNP and $300 \mathrm{ng} / \mathrm{L}$ for NT-proBNP, the NPs have sensitivities of 0.95 (95\% confidence interval $[\mathrm{CI}]$ 0.93-0.96) and 0.99 (95\% CI 0.97-1.00), and negative predictive values of 0.94 (95\% CI 0.90-0.96) and 0.98 (95\% CI 0.89-1.00), respectively, for a diagnosis of acute HF. Importantly, the authors did not observe a significant difference between BNP and NT-proBNP. Although this is the main study to support the recommendations in clinical guidelines, the cut-off proposed is for acute patients in the emergency room but this has been generalized to all HF patients, including outpatients. Indeed, the diagnostic thresholds vary depending on clinical characteristics due to the nature of the dynamic process of HF and the intra-/ interpatient variability in biomarkers measurements. For example, NP levels may be disproportionally low in obese patients, potentially leading to underdiagnosis in patients with high body mass index. ${ }^{15}$

Another important issue in HF clinical guidelines is related to the prediction of cardiac toxicity with cancer therapies. The use of NT-proBNP levels to assess structural cardiac involvement should be considered but it is not a substitute for cardiac evaluation with echocardiography. For that reason, clinical guidelines only suggest the use of biomarkers in cancer therapies with a weak recommendation. ${ }^{16}$

\section{Troponin for Diagnosis-Making Process in Chronic and Acute Heart Failure (Formal Clinical Guidelines Recommendations in - Table 1)}

\section{European Society of Cardiology Clinical Guidelines of $\mathrm{HF}^{5}$}

- Elevated concentrations of circulating cardiac troponins are detected in the vast majority of patients with acute HF, often without obvious myocardial ischemia or an acute coronary event, suggesting ongoing myocardial stress.

Comment to the Recommendations in Clinical Guidelines In the Atherosclerosis Risk in Communities (ARIC) study, the association of troponin $\mathrm{T}$ with incident coronary heart disease and subjects with high-sensitivity troponin T (cTnT) levels in the highest category had significantly increased risk for $\mathrm{HF}$ (hazard ratio [HR] 5.95; 95\% CI 4.47-7.92); of note, even minimally elevated $\mathrm{cTnT}(\geq 0.003 \mathrm{~g} / \mathrm{L})$ was associated with increased risk for mortality and HF $(p<0.05)$. Similar results regarding the troponin $\mathrm{T}$ levels related to mortality and poor prognosis were observed in the ADHERE study. ${ }^{11}$ Based on these results, clinical guidelines recommend routine ischemic assessment using high-sensitivity troponins at 
admission to rule-out concomitant coronary disease and as a biomarker of prognosis.

\section{Renal Function in Complete Management of HF Patients (Formal Clinical Guidelines Recommendations in - Table 1)}

\section{European Society of Cardiology Clinical Guidelines of $\mathrm{HF}^{5}$}

- HF and chronic kidney disease (CKD) frequently coexist, sharing many risk factors (diabetes, hypertension, and hyperlipidemia) and interacting to worsen prognosis.

Comment to the Clinical Guidelines Recommendations About one-third of HF patients have a concomitant mild or moderate CKD and about a quarter develop worsening renal function during their hospitalization for $\mathrm{HF} .{ }^{17}$ The worsening of renal function over the time in patients with HF has been associated with a reduction in survival and an increase of hospital admissions. Some of the deterioration may be related to diuretic therapy or with use of drugs such as the angiotensinconverting enzyme inhibitors, angiotensin receptor blockers, or aldosterone antagonist use. The worsening of heart pump function is also related to renal dysfunction ${ }^{17,18}$

\section{Other Biomarkers (Formal Clinical Guidelines Recommendations in - Table 1)}

Comment to the Recommendations in Clinical Guidelines In recent years, neurohormonal biomarkers and other biomarkers related to the inflammation and with remodeling/fibrosis have emerged. Despite this, clinical trials and observational registries are usually based only on a single measurement at baseline or study entry, and extrapolating their usefulness for treatment, monitoring, or as a prognosis markers should be taken with caution.

Neurohormonal activation has an important role in the progression and worsening of patients with $\mathrm{HF}$, for example, components of the renin angiotensin system (renin, angiotensin II, aldosterone), sympathetic nervous system (norepinephrine, chromogranin A, mid-regional proadrenomedullin), arginine vasopressin system (arginine vasopressin, copeptin), and endothelins (ET-1, big proET-1). ${ }^{5}$ In the Biomarkers in Acute Heart Failure (BACH) trial, Maisel et $\mathrm{al}^{15}$ analyzed the role of copeptin and other biomarkers in 557 patients with HF. Copeptin was highly prognostic for 90day adverse events in patients with acute HF, adding prognostic value to clinical predictors, serum sodium, and NPs; however, the potential variability and the influence in plasma levels due to the different laboratory tests and patient clinical stability limits the generalization of these results.

Related to cardiac remodeling and fibrosis, the measurement of soluble suppression of tumorigenicity 2 (sST2) is correlated to LV hypertrophy, fibrosis, and remodeling via interaction with interleukin (IL)-33, a protein with antifibrotic and antiremodeling. ${ }^{19}$ In one study, Ky et al $^{20}$ determined whether plasma SST2 levels predict adverse outcomes in 1,141 patients with chronic HF and observed those with the highest sST2 tertile (sST2 $>36.3 \mathrm{ng} / \mathrm{mL}$ ) had a markedly increased risk of adverse outcomes compared with the lowest tertile (sST2 $\leq 22.3 \mathrm{ng} / \mathrm{mL}$ ), even after multivariable adjustment (HR 1.9; 95\% CI, 1.3-2.9; $p=0.002$ ).

Galectin-3 (Gal-3) is another important biomarker related to cardiac fibrosis. In the DEAL-HF study, Lok et $\mathrm{al}^{21}$ analyzed the role of Gal-3 levels in cardiac remodeling in HF and concluded that this biomarker was a significant predictor of mortality risk, even after adjustment for age and sex. Indeed, its prognostic value was independent of severity of HF and renal dysfunction, as assessed by NT-proBNP and estimated glomerular filtration rate (eGFR), respectively.

The use of these potential novel biomarkers for daily clinical practice is controversial. One limitation is the small sample size of many (often heterogeneous) studies, as well as the potential variability of the biomarker over time and the status of the HF patient at the moment of the measurement. Indeed, the predictive ability that offers the inclusion of biomarkers for prognosis of HF is modest. Also, the exclusion of the influence of other biomarkers that could act as confounding factors is not always controlled. For that reason, recommendations for routine use of other nonstandardized biomarkers (copeptin, sST2, or Gal-3) are weak and limited to specific clinical situations. For example, increased Gal-3 was a proportional predictor of CV death and all-cause mortality, also after adjustment for NTproBNP, in subjects without previously diagnosed CVD. ${ }^{22}$

\section{Biomarkers in Atrial Fibrillation}

Atrial fibrillation (AF) is associated with high morbidity and mortality, largely attributable to an increased risk of stroke and thromboembolism. Oral anticoagulation treatment is highly effective in reducing the risk of stroke by $64 \%$ and all-cause mortality by $26 \%$ in comparison with placebo or control. ${ }^{23}$

Biomarkers such as markers of inflammation, coagulation activity, CV stress, myocardial injury, and cardiac and renal dysfunction have all shown high association with clinical events and have been proposed to refine risk assessment in AF patients. ${ }^{24}$ Cardiac biomarkers, such as troponin and NPs, also significantly improve risk stratification in addition to current clinical risk stratification models.

The use of biomarkers in AF helps in the understanding of the pathophysiology of this prevalent disease and could refine stroke and major bleeding risk in AF patients. ${ }^{25}$ Indeed, most of the clinical guidelines recommendations are only weak, based on current evidence and expert consensus documents. Any biomarker, whether blood, urine, or imaging (cardiac, cerebral, or otherwise), will always improve on risk prediction based on clinical factors, but this needs to be balanced against the practical usefulness, cost, and daily applicability for everyday clinical practice. ${ }^{26}$

\section{Pathophysiology of Atrial Fibrillation}

2016 European Society of Cardiology Clinical Guidelines of Atrial Fibrillation ${ }^{27}$

- Activation of fibroblasts, enhanced connective tissue deposition, and fibrosis are the hallmarks of structural 
remodeling in the atria. Atrial fatty infiltration, inflammatory infiltrates, myocyte hypertrophy, necrosis, and amyloidosis are found in $\mathrm{AF}$ patients with concomitant conditions predisposing to AF.

- Although biomarkers such as NPs are elevated in AF patients, there is insufficient data to suggest that bloodbased parameters are independent markers for AF.

2014 AHA/ACC/HRS Guideline for the Management of Patients with Atrial Fibrillation 28

- Multiple clinical risk factors, electrocardiographic and echocardiographic features, and biochemical markers are associated with an increased risk of AF.

Antithrombotic Therapy for Atrial Fibrillation: American College of Chest Physicians Guidelines and Expert Panel ReportACCP $2018^{26}$

- The use of biomarkers includes inter- and intrapatient and assay variability, some have a diurnal variation and can be highly influenced by associated comorbidities and drug therapies.

Comment on Recommendations in the Clinical Guidelines Different biomarkers have been proposed to predict patients at risk of AF development. An inflammatory state is related to AF burden and atrial remodeling. IL-6 and C-reactive protein (CRP) are robust and established markers of inflammation and have been most frequently investigated in CVD and AF.
Aviles et al $^{29}$ analyzed the influence of CRP levels in 5,806 patients enrolled in the Cardiovascular Health Study with 7 years of follow-up, and concluded that elevated baseline CRP levels predicted an increased risk for developing future AF. Similarly, Patton et al $^{30}$ analyzed the role of NT-proBNP in the same registry and observed that this biomarker was also an independent predictor of AF, even after adjustment for an extensive number of covariates; also NT-proBNP levels were also strongly associated with prevalent AF. Recently, Chua et $\mathrm{al}^{31}$ showed in 648 patients that elevated levels of BNP (odds ratio [OR] 1.29, 95\% CI 1.11-1.63; $p=0.002$ ) and elevated levels of fibroblast growth factor-23 (OR 1.67, 95\% CI $1.36-2.34 ; p=0.001$ ) were significantly associated with prevalent AF.

However, many of the published studies generally analyzed the effect of one (bio)marker in the time and the pathophysiology of AF development is a continuum.

\section{Stroke Risk Assessment in Atrial Fibrillation Patients (Formal Clinical Guidelines Recommendations in - Table 2)}

\section{European Society of Cardiology Clinical Guidelines of} Atrial Fibrillation ${ }^{27}$

- Biomarker-based risk scores may, in the future, be helpful to better stratify patients (e.g., those at a truly low risk of stroke).

Table 2 Main current clinical guidelines recommendations for biomarkers use in atrial fibrillation

\begin{tabular}{|c|c|c|c|}
\hline & ESC (27) & $\mathrm{AHA} / \mathrm{ACC}(64)$ & $\begin{array}{l}\text { American College of Chest } \\
\text { Physicians Guidelines-2018 ACCP (26) }\end{array}$ \\
\hline $\begin{array}{l}\text { Atrial } \\
\text { fibrillation }\end{array}$ & $\begin{array}{l}\text { - Measurement of cardiac tro- } \\
\text { ponin and NT-proBNP may } \\
\text { provide additional prognostic } \\
\text { information in selected AF } \\
\text { patients. } \\
\text { - Biomarkers such as Hs-troponin } \\
\text { and NPs may be considered to } \\
\text { refine stroke and bleeding risk } \\
\text { Class Ilb, level of evidence B } \\
\text { - Several bleeding risk scores } \\
\text { have been developed, mainly in } \\
\text { patients on VKAs. These in- } \\
\text { clude HAS-BLED, ORBIT, and } \\
\text { more recently, the ABC bleed- } \\
\text { ing score, which also makes use } \\
\text { of selected biomarkers } \\
\text { - The assessment of kidney } \\
\text { function by serum creatinine or } \\
\text { creatinine clearance is recom- } \\
\text { mended in all AF patients } \\
\text { Class I, level of evidence A }\end{array}$ & $\begin{array}{l}\text { - Renal function and hepatic } \\
\text { function should be evaluated } \\
\text { before initiation of a NOAC and } \\
\text { should be reevaluated at least } \\
\text { annually } \\
\text { Class I, level of evidence: B }\end{array}$ & $\begin{array}{l}\text { - Many biomarkers are also predic- } \\
\text { tive of stroke, bleeding, death, } \\
\text { heart failure, and hospitalization in } \\
\text { AF patients } \\
\text { - Current studies do not inform } \\
\text { whether biomarkers will } \\
\text { discriminate/identify low risk in } \\
\text { lower/intermediate risk patients } \\
\text { who are not anticoagulated } \\
\text { - The addition of biomarkers to } \\
\text { bleeding risk scores would all } \\
\text { improve the c-indexes (to approx. } \\
\text { 0.65) over scores based on clinical } \\
\text { risk factors alone but marginally. The } \\
\text { performance of biomarker-based } \\
\text { scores in real world clinical practice } \\
\text { (outside highly selected trial cohorts) } \\
\text { has also been disappointing } \\
\text { - CKD is an independent predictor of } \\
\text { risk of stroke/thromboembolism. } \\
\text { The clinical relevance of renal func- } \\
\text { tion is not only for risk prediction, but } \\
\text { also for choice of anticoagulation and } \\
\text { other therapies }\end{array}$ \\
\hline
\end{tabular}

Abbreviations: ACC, American College of Cardiology; AF, atrial fibrillation; AHA, American Heart Association; CKD, chronic kidney disease; ESC, European Society of Cardiology; NT-proBNP, N-terminal pro-B-type natriuretic peptide; VKAs, vitamin K antagonists. 
Antithrombotic Therapy for Atrial Fibrillation: American College of Chest Physicians Guidelines and Expert Panel ReportACCP $2018^{26}$

- The importance of biomarkers probably lies in the "very low risk" strata of clinical scores (e.g., $\mathrm{CHA}_{2} \mathrm{DS}_{2}$-VASc $=0$ 1 group) where they may influence the decision to anticoagulate. For these reasons, the clinical application of biomarkers in management of $\mathrm{AF}$ is unlikely to be significant.

- Current studies do not inform whether biomarkers will discriminate/identify low risk in lower/intermediate risk patients who are not anticoagulated.

\section{Comment to the Clinical Guidelines Recommendations}

Stroke and thromboembolic risk in AF patients is assessed using $\mathrm{CHA}_{2} \mathrm{DS}_{2}$-VASc risk score. ${ }^{26,32,33}$ This score has only clinical and demographic variables and has modest ability to predict thromboembolic events and mortality. ${ }^{34,35}$ Biomarkers were first proposed to refine clinical risk stratification $>10$ years ago. ${ }^{36}$ Lip et $\mathrm{al}^{36}$ analyzed the role of von Willebrand factor (vWF) (as plasma biomarker of endothelial damage/dysfunction and associated with stroke risk) to refine stroke prevention in AF patients. The authors observed that the addition of plasma vWF levels refined stroke risk stratification in $\mathrm{CHADS}_{2}$ score, particularly in AF patients at moderate risk with the corresponding c-indexes for ischemic stroke and vascular events of 0.691 (95\% CI, 0.600-0.772) and 0.697 (95\% CI, 0.626-0.763), respectively. Indeed, high vWF levels were independently associated with a higher risk of vascular events.

In recent years, there has been renewed interest in these biomarkers which have been part of ancillary analyses to large randomized controlled trials. These biomarkers are related to myocardial stress (troponin or NT-proBNP), renal function (cystatin C), the prothrombotic state in AF patients (D-dimer, vWF, soluble thrombomodulin, or soluble E-selectin), or the inflammatory state (CRP or IL-6). ${ }^{37}$ Recently, other oxidative stress markers have also been related with adverse events in $\mathrm{AF}$ patients. Oxidative stress has been shown to promote and maintain $\mathrm{AF}$ with a mechanism related to upregulation of myeloperoxidase (MPO) and NOX2. Indeed, oxidative stress is implicated in clotting activation. ${ }^{38}$ The urinary excretion of F2IsoP (a particular type of prostaglandin) have been related with thrombosis and systemic atherosclerosis. ${ }^{39}$ Pignatelli et $\mathrm{al}^{40}$ analyzed the effect of oxidative biomarkers (serum NOX2 and urinary isoprostanes), in AF patients and observed that high levels of these biomarkers were related with adverse events and mortality. The addition of tertiles of urinary isoprostanes significantly increased the predictive performance and net reclassification index of $\mathrm{CHA}_{2} \mathrm{DS}_{2}$-VASc for $\mathrm{CV}$ events and mortality.

The use of biomarkers (whether urine, blood, or imaging) adds statistical improvement in the predictive performance of risk scores compared to schemes using clinical variables, approximately 0.65 to 0.70 . The use of biomarkers should balance the assay availability, laboratory variability, costs and added complexity, and lower practicality for everyday use. Also, many biomarker studies are based on anticoagu- lated highly selected clinical trial cohorts. ${ }^{26}$ For that reason, the minimal improvement and complexity of use makes the applicability in daily clinical practice doubtful.

Related to myocardial damage, minor elevations of troponin below the 99th percentile of the upper reference limit (URL) (to diagnosis of myocardial infarction [MI]) have been observed in patients with $\mathrm{AF}$, in most cases due to rapid and irregular ventricular response in AF patients. Van den Bos et $\mathrm{al}^{41}$ conducted a prospective study with consecutive AF patients, excluding patients with MI, and observed that minor elevations in troponin I during hospital admission had an independent association with mortality (HR 2.35; 95\% CI 1.17-4.73] and major adverse cardiovascular events (MACEs) (HR 2.48; 95\% CI 1.33-4.63).

Roldán et $\mathrm{al}^{42}$ analyzed the role of cTnT in $930 \mathrm{AF}$ patients with stable oral anticoagulation. In this study, median (interquartile range) values of hsTnT were $8.86(4.24-15.21) \mathrm{pg} / \mathrm{mL}$, and 291 (31\%) patients had hsTnT levels above $13 \mathrm{pg} / \mathrm{mL}$, that is the 99th percentile of hsTnT and the cut-point proposed for the diagnosis of MI. The authors found that high levels of troponin (above $8.04 \mathrm{pg} / \mathrm{mL}$ ) were significantly associated with longterm adverse CV events, even after adjusting for the $\mathrm{CHADS}_{2}$ score (HR 2.21; 95\% CI 1.46-3.35, $p<0.001$ ).

Abnormal levels of troponins are related not only with mortality and MI in AF patients but also to stroke and thromboembolic risk. The role of cardiac biomarkers was also analyzed in a biomarker substudy of 6,189 AF patients from Randomized Evaluation of Long Term Anticoagulant Therapy (RE-LY), ${ }^{43}$ where troponin I was significantly and independently associated with increased risk of stroke or systemic embolism. The annual rates of stroke or systemic embolism were lowest, being $0.84 \%$, in the group with undetectable troponin I, which is in comparison with 2.09\% (HR, 1.99; 95\% CI, 1.17-3.39) in the highest troponin I group $(p=0.0040)$. The addition of troponin I and NT-proBNP significantly improved the predictive performance of $\mathrm{CHADS}_{2}$ for stroke outcomes. Note that the improved c-statistics remained $<0.7$ despite the addition of two biomarkers, in this highly selected anticoagulated clinical trial cohort. In a similar study, the Apixaban for the Prevention of Stroke in Subjects with Atrial Fibrillation (ARISTOTLE) troponin substudy analyzed 14,892 AF patients and also observed that troponin T levels were independently associated with an increased risk of stroke, cardiac death, and major bleeding and improved risk stratification beyond the $\mathrm{CHA}_{2} \mathrm{DS}_{2}$-VASc risk score. ${ }^{44}$

Other important biomarkers analyzed are NPs. The relationship between NPs levels and adverse outcomes in AF patients was assessed in the RE-LY trial. ${ }^{43} \mathrm{NT}$-proBNP levels were positively correlated with the risk of thromboembolic events and CV mortality, with higher risk at rising levels. Indeed, the addition of NT-proBNP to the $\mathrm{CHADS}_{2}$ and $\mathrm{CHA}_{2} \mathrm{DS}_{2}$-VASc risk stratification models resulted in significant improvements in the discrimination performance for both outcomes as well. A "real-world" study conducted by Roldán et $\mathrm{al}^{45}$ observed that NT-proBNP provided complementary prognostic information to an established clinical risk score $\left(\mathrm{CHA}_{2} \mathrm{DS}_{2}\right.$-VASc) for the prediction of stroke/ systemic embolism and all-cause mortality. 
Based on adding biomarkers to ischemic risk scores in AF patients, a new thromboembolic risk score has emerged, the ABC (age, cardiac biomarkers [NT-proBNP, high-sensitivity cardiac troponin], and clinical history [prior stroke/transient ischemic attack])-stroke risk score. The ABC-stroke score was derived and validated in two clinical trial cohorts (14,701 AF patients) in which patients with AF are often highly selected and carefully followed-up. ${ }^{46}$ Compared with the widely used clinically based $\mathrm{CHA}_{2} \mathrm{DS}_{2}$-VASc score, the $\mathrm{ABC}$-stroke risk score had better predictive performance for adverse events. However, the results of the $\mathrm{ABC}$ score in several independent real-world cohorts have been disappointing. ${ }^{47,48}$ For example, Rivera-Caravaca et al $^{47}$ validated the $\mathrm{ABC}$-stroke in $\mathrm{AF}$ patients under stable anticoagulation with acenocoumarol, over a long-term period of follow-up (6.5 years); they found that the ABC-stroke score did not offer significantly better predictive performance compared with the $\mathrm{CHA}_{2} \mathrm{DS}_{2}$-VASc score.

All these clinical studies analyzed the potential role of biomarkers for stroke prediction in AF patients and could help to better understand the different mechanisms involved in thromboembolism events in AF patients and guide the therapy in some selected patients. However, the recent realworld studies have only shown a slight improvement in the predictive performance with the use of biomarkers (c-statistics generally remain $<0.7$ despite biomarkers), and waiting for biomarker test results could delay the initiation of oral anticoagulation therapy (-Table 3 ).

\section{Bleeding Risk Assessment in Atrial Fibrillation Patients (Formal Clinical Guidelines Recommendations in - Table 2)}

Antithrombotic Therapy for Atrial Fibrillation: American College of Chest Physicians Guidelines and Expert Panel Report. ACCP $2018^{26}$

- Many biomarkers are nonspecific for a particular endpoint, and can be equally predictive not only for stroke but bleeding, death, hospitalization, HF, etc., as well as noncardiac conditions, for example, glaucoma (growth differentiation factor-15 [GDF-15]).

- The performance of biomarker-based scores in real-world clinical practice (outside highly selected trial cohorts) has also been disappointing, given that baseline (or nearbaseline) determination of biomarkers to predict bleeding risks after many years is bedeviled by the changing clinical risk profile of patient's risks as well as modification of risk factors.

Comment to the Clinical Guidelines Recommendations In recent years, different bleeding risk scores have been proposed, some quite complex, to assess hemorrhagic risk in $\mathrm{AF}$ patients. The HAS-BLED bleeding score has been proposed as the main clinical score to assess major bleeding events, and is supported by a systematic review from the Patient-Centered Outcomes Research Institute(PCORI) where 38 studies explored bleeding risk scores and the HAS-BLED score provides the best prediction for bleeding risk and intracranial hemorrhage.
The HAS-BLED score has been validated in AF patients with and without anticoagulation therapy and it is the only bleeding score for intracranial hemorrhage prediction. ${ }^{49,50}$ The most common bleeding risk scores (HAEMORR ${ }_{2}$ HAGES, HAS-BLED ATRIA, ORBIT) mainly include risk factors and clinical variables that provide only modest predictive value for predicting patients at high risk of bleeding, and generally underperform compared to HAS-BLED in vitamin K antagonist (VKA)-treated patients as they neglect to consider quality of anticoagulation control. ${ }^{48,51,52}$ Indeed, these bleeding risk scores have been validated also in patients under venous thromboembolism. ${ }^{53}$

Different biomarkers emerged to explain the pathophysiology of bleeding events in AF patients and have been used to refine bleeding risk prediction in AF patients. Roldán et $\mathrm{al}^{54}$ reported the use of vWF levels to predict bleeding events and observed that high plasma vWF levels ( $\geq 221 \mathrm{IU} / \mathrm{dL}$ ) are an independent risk factor for major bleeding and mortality in anticoagulated AF patients; but this biomarker was also predictive of stroke.

In a substudy of the RE-LY trial, Hijazi et al analyzed the role of troponin T and NT-proBNP for bleeding risk assessment ${ }^{43}$ and reported an association between elevated troponin I levels and the risk of major bleeding. However, there was no significant association between NT-proBNP levels and major bleeding. ${ }^{43,55}$

Recently, a new biomarker related to bleeding in anticoagulated AF patients has been proposed, GDF-15. This biomarker is a marker of oxidative stress and inflammation, providing independent prognosis information on CV events beyond $\mathrm{CV}$ risk factors and other biomarkers. Wallentin et $\mathrm{al}^{56}$ analyzed the role of GDF-15 in a substudy of the ARISTOTLE clinical trial and observed that this biomarker was an independent biomarker for major bleeding in AF patients. Higher GDF-15 levels were significantly associated with a 3.5-fold higher rate of major bleeding and the addition of GDF-15 to the HAS-BLED and ORBIT bleeding scores significantly improved the predictive performance of the clinical factor-based scores for major bleeding. ${ }^{57}$

However, GDF-15 is not a specific biomarker of bleeding risk and several conditions could modify the plasma levels of this biomarker, being an oxidative stress marker. For example, Sharma et $\mathrm{al}^{58}$ analyzed the role of GDF-15 levels in HF patients enrolled in the HF-ACTION (Heart Failure: A Controlled Trial Investigating Outcomes of Exercise Training) trial. The authors observed that high concentration of GDF15 was related with a $30 \%$ greater risk of mortality and with worse symptom burden and low functional capacity in stable HF patients. However, GDF-15 biomarker is not only related to adverse outcomes in CVD patients but also seems predictive of non-CVD conditions, such as glaucoma progression. ${ }^{59}$ Indeed, GDF-15 acts as a molecular marker that predicts glaucomatous neurodegeneration, and elevated levels of GDF-15 were significantly associated with worse functional outcomes in glaucoma patients.

Based on these biomarkers tested in AF, the ABC-bleeding score Age [A], Biomarkers (cTn-hs, hemoglobin, GDF-15 or creatinine clearance $[\mathrm{CrCl}]$ ) $[\mathrm{B}]$, and clinical history (previous 


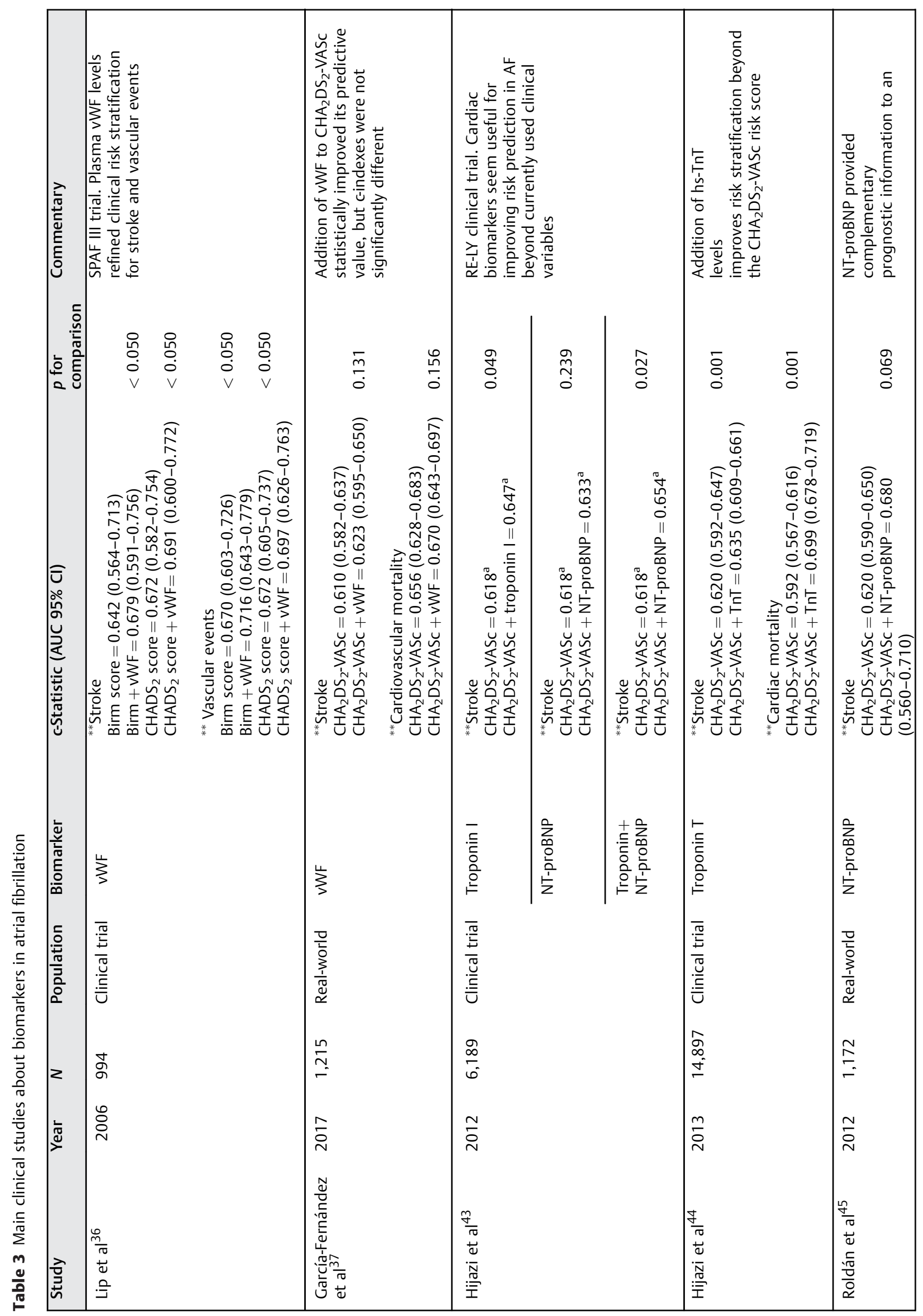




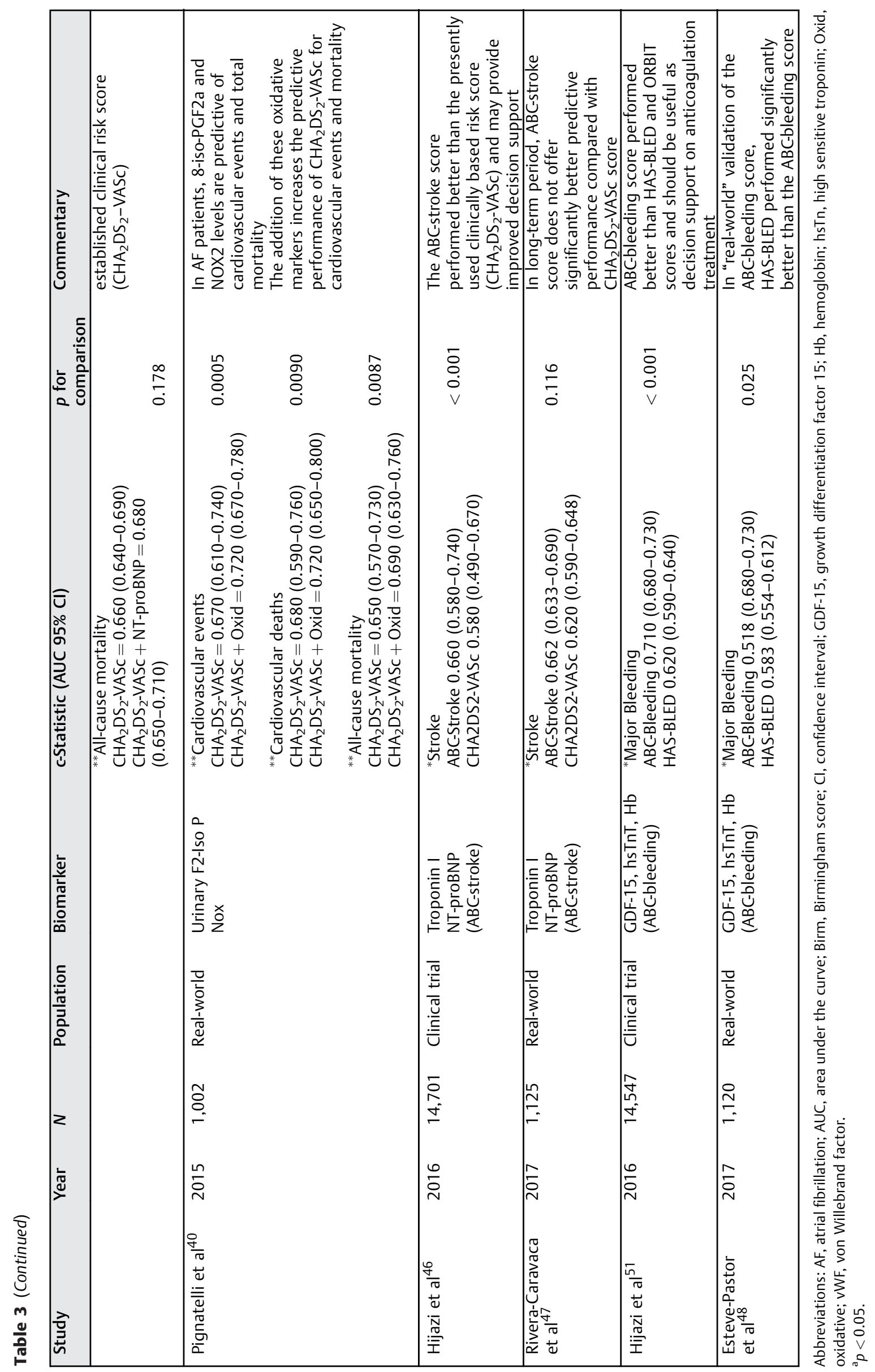


bleeding) [C] was developed from the ARISTOTLE clinical trial and was externally validated in the RE-LY trial. ${ }^{51,57,60}$ The ABC-bleeding score performed better than HAS-BLED score and was proposed as useful tool to support decision-making for anticoagulation treatment in AF patients. ${ }^{51}$ The original validation of $A B C$-bleeding score included GFD-15, cTn-hs, and hemoglobin, but Hijazi et al also proposed a modified ABC-bleeding score using alterative biomarkers (cTn-hs, hemoglobin, and $\mathrm{CrCl}$ ) instead of GDF-15; however, the use of GDF-15 has been only validated in anticoagulated patients (with non-VKAs oral anticoagulants [NOACs] or VKAs) but there are no data available in AF patients without anticoagulation.

An independent real-world external validation analyzed the predictive performance of ABC-bleeding in a nonselected AF population under VKAs. ${ }^{48}$ Using sensitivity, reclassification, and decision curve analyses, the authors concluded that the HAS-BLED score performed significantly better than the $A B C$-bleeding score in predicting major bleeding, gastrointestinal bleeding, and intracranial hemorrhage. These results highlight the difference between real-world and clinical trials, since patients from clinical trials are carefully selected with a close follow-up. Indeed, real-world patients are older, with multiple comorbidities, and polypharmacy that could influence the levels and variability of blood biomarkers.

In this sense, a recent study analyzed the adverse events between real-world AF cohort and the AMADEUS clinical trial cohort and concluded that there was great heterogeneity in both populations, which is translated into a higher risk of several adverse outcomes in a real-world cohort, including major bleeding, ischemic stroke, and mortality. ${ }^{61}$

Biomarkers might be helpful in assessing the risk of major bleeding, providing information in complex cases to select the best net clinical benefit therapy for the patients. Nevertheless, the addition of biomarkers to ischemic and bleeding risk scores only modestly improves their predictive performance, with no clinical difference when compared to clinical risk scores. ${ }^{32}$ Indeed, bleeding risk assessment is a dynamic process that needs regular evaluation and review. ${ }^{62} \mathrm{~A}$ dynamic and practical score is mandatory with modifiable variables to highlight patients potentially at high risk of bleeding to address the reversible bleeding risk factors.

The biological variability of blood or urine biomarkers has only had limited attention in the clinical trials. Some biomarkers have very large diurnal variations and, for that reason, may not be useful as single time-point measurement for patients in the anticoagulation clinics or in the ward to decide oral anticoagulation process. Indeed, a range of biomarker levels between normal controls and sick patients is mandatory but depends on age, sex, and other related comorbidities. ${ }^{63}$ Nowadays, it is still very difficult to find a biomarker with little variability throughout the day, with high quality and precision to select healthy and sick patients and high specificity for each pathology. Also in this way, a bedside simple bleeding score for daily clinical practice is necessary and the inclusion of routine biomarkers in risk scores implies losing simplicity and practicality. For that reason, current clinical guidelines only provide weak recom- mendation to the role of biomarkers for stroke and bleeding risk assessment in AF patients.

\section{Renal Function in Complete Management of AF Patients (Formal Clinical Guidelines Recommendations in - Table 2)}

2016 European Society of Cardiology Clinical Guidelines of Atrial Fibrillation ${ }^{27}$

- AF is present in 15 to $20 \%$ of patients with CKD. The definition of CKD in most AF trials is relatively strict. Although an estimated $\mathrm{CrCl}$ rate of $60 \mathrm{~mL} / \mathrm{min}$ is indicative of $\mathrm{CKD}$, a number of trials in $\mathrm{AF}$ patients have used $\mathrm{CrCl}$ $50 \mathrm{~mL} / \mathrm{min}$ to adapt NOAC dosage, usually estimated using the Cockroft-Gault formula. $\mathrm{CrCl}$ in $\mathrm{AF}$ patients can deteriorate over time.

- All AF patients treated with oral anticoagulation should be considered for at least yearly renal function evaluation to detect CKD (Class Ila, Level of Evidence B).

2019 AHA/ACC/HRS Focused Update of the 2014 AHA/ACC/ HRS Guideline for the Management of Patients with Atrial Fibrillation $^{64}$

- Renal function and hepatic function should be evaluated before initiation of a NOAC and should be reevaluated at least annually.

- All 4 NOACs with FDA approval for use in patients with AF have dosing defined by renal function (creatinine or $\mathrm{CrCl}$ using the Cockcroft-Gault equation).

- Renal function should be regularly monitored and $\mathrm{CrCl}$ calculated at an interval that depends on the individual degree of renal dysfunction and likelihood of fluctuation, and dose adjustments should be made according to the FDA dosing guidelines.

Antithrombotic Therapy for Atrial Fibrillation: American College of Chest Physicians Guidelines and Expert Panel Report ACCP $2018^{26}$

- AF patients with estimated glomerular filtration rate $<60$ $\mathrm{mL} / \mathrm{min}$ compared to those with estimated glomerular filtration rate $\geq 60 \mathrm{~mL} / \mathrm{min}$ have increased risk of stroke/ thromboembolism (relative risk [RR] 1.62, 95\% CI, 1.40$1.87 ; p<0.001)$, with a $0.41 \%(0.17-0.65 \%)$ annual rate increase for every $10 \mathrm{~mL} / \mathrm{min}$ decrease in renal function. The risk is higher in individuals requiring renal replacement therapy (HR 1.83; 95\% CI, 1.57-2.14; $p<0.001$ ).

Comment on Recommendations in the Clinical Guidelines CKD is highly prevalent in AF patients and both renal dysfunction and AF are independently associated with a higher risk of mortality and thromboembolic and bleeding events. A recent study from the prospective FANTASIIA registry ${ }^{65}$ observed that renal function was independently related to CV mortality, major bleeding, and MACE but not to thromboembolic events. Patients with severe CKD have a fivefold risk of major bleeding compared to those with normal renal function and renal dysfunction is one of the components of the HAS-BLED score. 
Another study published by Roldán et al $^{66}$ showed that the addition of CKD to stroke risk scores (CHADS $_{2}$ and $\mathrm{CHA}_{2} \mathrm{DS}_{2}$-VASc) did not independently improve the predictive ability of current clinical scores. Bonde et $\mathrm{al}^{67}$ demonstrated in 154,259 AF patients that CKD is associated with a higher risk of stroke/thromboembolism across stroke risk strata in AF patients. Of note, high-risk patients $\left(\mathrm{CHA}_{2} \mathrm{DS}_{2}-\right.$ VASc $\geq 2$ ) with CKD had benefit with warfarin treatment for stroke prevention. In the same way, the use of NOACs was associated with a reduced risk of stroke and major bleeding compared to warfarin in patients with renal disease, with positive net clinical benefit. ${ }^{68}$ Dosing recommendations for patients with renal impairment differ depending on the NOAC, whereby some of the NOACs require dose reductions based solely on renal function, while others require consideration of additional criteria. For that reason, renal function should be evaluated in patients on a NOAC, as worsening of renal function may warrant change in the dose of a NOAC or change in oral anticoagulant.

Despite it seems mandatory to assess renal function at baseline, it is also important to monitor renal function over time. Roldán et $\mathrm{al}^{69}$ and FANTASIIA registry investigators ${ }^{66}$ demonstrated that worsening glomerular filtrated rate (eGFR) of only $\geq 10 \mathrm{~mL} / \mathrm{min}$ during follow-up was significantly associated with mortality and major bleeding. Fauchier et $\mathrm{al}^{70}$ showed that worsening in eGFR is an independent predictor of ischemic stroke, thromboembolism, and bleeding in AF patients.

For that reason, clinical guidelines recommend assessing and monitoring renal function at least yearly in AF patients under oral anticoagulation with strong evidence.

\section{Biomarkers in Coronary Artery Disease}

MI is defined by clinical history, ECG, and increased levels of cardiac troponins as biomarkers reflecting myocardial damage. ${ }^{71}$ The role of biomarkers in the definition and diagnosis of ACS is essential and different clinical guidelines reflect this point. Formal clinical guidelines recommendations in - Table 4.

Fourth Universal Definition of Myocardial Infarction $(2018)^{71}$

- The myocardial injury is considered acute if there is a rise and/or fall of cardiac troponin values.

2017 ESC Guidelines for the Management of Acute Myocardial Infarction in Patients Presenting with ST-Segment Elevation ${ }^{72}$

- The term acute myocardial infarction (AMI) should be used when there is evidence of myocardial injury (defined as an elevation of cardiac troponin values with at least one value above the 99th percentile URL) with necrosis in a clinical setting consistent with myocardial ischemia.

2014 AHA/ACC Guideline for the Management of Patients with Non-ST-Elevation Acute Coronary Syndromes $(\text { NSTEMI) })^{73}$

- If the time of symptom onset is ambiguous, the time of presentation should be considered as the time of onset for assessing troponin values (Class I, Level of Evidence: A).
- Regarding the use of biomarkers for prognosis: the presence and magnitude of troponin elevations are useful for shortand long-term prognosis (Class I, Level of Evidence B).

- It may be reasonable to remeasure troponin once on day 3 or day 4 in patients with a MI as an index of infarct size and dynamics of necrosis (Class IIb, Level of Evidence B).

- The use of selected newer biomarkers, especially BNP, may be reasonable to provide additional prognostic information.

2015 ESC Guidelines for the Management of Acute Coronary Syndromes in Patients Presenting without Persistent ST-Segment Elevation ${ }^{74}$

- A rapid rule-out and rule-in protocol at 0 and 1 hour is recommended if a hs-cTn test with a validated $0 \mathrm{~h} / 1 \mathrm{~h}$ algorithm is available. Additional testing after 3 to 6 hours is indicated if the first two troponin measurements are not conclusive and the clinical condition is still suggestive of ACS (Class I, Level of Evidence A).

- Beyond diagnostic utility, cardiac troponin levels add prognostic information in terms of short- and long-term mortality to clinical and ECG variables. The extensively validated NPs (i.e., BNP, NT-proBNP, and mid-regional pro-A-type NP) provide prognostic information on top of cardiac troponin.

Comment Recommendations in the Clinical Guidelines Cardiac biomarkers have an essential role in the "rule-in" and "rule-out" of ACS in patients with chest pain. Biomarkers complement clinical assessment and the 12-lead ECG in the diagnosis, risk stratification, triage, and management of patients with suspected ACS. The measurement of cardiac injury biomarkers shows the magnitude of the damage and it is a prognostic marker.

Different studies have demonstrated the value of highsensitive troponins (hs-cTn) to increase the accuracy of AMI diagnosis and the benefit of hs-cTn compared with nonsensitive assays was more pronounced in patients presenting early at emergency room after chest pain. ${ }^{75}$ Reichlin et al ${ }^{76}$ analyzed the role of sensitive cardiac troponin assays in the emergency department for ACS diagnosis and found the predictive performance of hs-cTnT and hs-cTnI was high (c-statistics between 0.96 and 0.92 , respectively) for final diagnosis of AMI. These results were supported by a multicentric prospective study using four different brands of hs-cTns to assess the negative predictive value of troponins to rule out AMI in patients with chest pain and concluded that undetectable levels of hs-cTns at presentation had a very high negative predictive value and seem to allow the simple and rapid rule out of AMI. Recently, the algorithm 0/1 hour for rapid rule-out and rule-in of NSTEMI using hs-cTns has been validated by the European Society of Cardiology (ESC). Biomarkers were assessed at entry and after 1 hour of admission. Using 4,368 patients with serial cTnT measurements and 3,500 patients with serial cTnI measurements, this algorithm was validated to be very safe and effective in triaging patients with suspected NSETMI. ${ }^{77}$ However, the results of blood biomarkers in STEMI patients in any case could lead to a delay in revascularization. ${ }^{72}$ Other biomarkers like creatinine kinase MB isoform have demonstrated less sensitivity and less specificity for 
Table 4 Main current clinical guidelines recommendations for biomarkers use in coronary artery disease

\begin{tabular}{|c|c|c|c|}
\hline & $\operatorname{ESC}(72,74)$ & AHA/ACC (73) & $\begin{array}{l}\text { Fourth Universal Definition of } \\
\text { MI (71) }\end{array}$ \\
\hline $\begin{array}{l}\text { Coronary } \\
\text { artery } \\
\text { disease }\end{array}$ & $\begin{array}{l}\text { - Routine blood sampling for } \\
\text { serum markers is indicated as } \\
\text { soon as possible in the acute } \\
\text { phase but should not delay re- } \\
\text { perfusion treatment } \\
\text { Class I, level of } \\
\text { recommendation A } \\
\text { - Measurement of a biomarker of } \\
\text { cardiomyocyte injury, preferably } \\
\text { hs-cardiac troponin, is mandatory } \\
\text { in all patients with suspected } \\
\text { NSTEMI. It is recommended to } \\
\text { measure cardiac troponins with } \\
\text { sensitive or high-sensitivity assays } \\
\text { and obtains the results within } \\
60 \text { min. Additional testing after } \\
\text { 3-6 hours is indicated if the first } \\
\text { two troponin measurements are } \\
\text { not conclusive and the clinical } \\
\text { condition is still suggestive of ACS } \\
\text { Class I, level or } \\
\text { recommendation A } \\
\text { - GRACE risk score provides the } \\
\text { most accurate stratification of risk } \\
\text { both on admission and at dis- } \\
\text { charge in ACS. This risk score } \\
\text { includes serum creatinine values } \\
\text { and elevated cardiac biomarkers } \\
\text { Class I, level of evidence B }\end{array}$ & $\begin{array}{l}\text { - Cardiac-specific troponin (tropo- } \\
\text { nin I or T when a contemporary } \\
\text { assay is used) levels should be } \\
\text { measured at presentation and } 3 \\
\text { to } 6 \text { hours after symptom onset } \\
\text { in all patients who present with } \\
\text { symptoms consistent with ACS } \\
\text { Class I, level of evidence: A } \\
\text { - Additional troponin levels should } \\
\text { be obtained beyond } 6 \text { hours after } \\
\text { symptom onset in patients with } \\
\text { normal troponins on serial ex- } \\
\text { amination when electrocar- } \\
\text { diographic changes and/or } \\
\text { clinical presentation confer an } \\
\text { intermediate or high index of } \\
\text { suspicion for ACS } \\
\text { Class I, level of evidence: A } \\
\text { Creatine kinase myocardial isoen- } \\
\text { zyme (CK-MB) and myoglobin are } \\
\text { not useful for diagnosis of ACS } \\
\text { Class III, Level of Evidence A }\end{array}$ & $\begin{array}{l}\text { - The major criteria for myocardial } \\
\text { injury should be used when there } \\
\text { is evidence of elevated cardiac } \\
\text { troponin values with at least one } \\
\text { value above the } 99 \text { th } \\
\text { - The clinical definition of MI } \\
\text { denotes the presence of acute } \\
\text { myocardial injury detected by } \\
\text { abnormal cardiac biomarkers in } \\
\text { the setting of evidence of acute } \\
\text { myocardial ischemia }\end{array}$ \\
\hline
\end{tabular}

Abbreviations: ACC, American College of Cardiology; ACS, acute coronary syndrome; AHA, American Heart Association; ESC, European Society of Cardiology; Hs, high sensitive; MI, myocardial infarction; NSTEMI, non-ST elevation myocardial infarction.

myocardial injury. In the ESC guidelines for the fourth definition of MI, myocardial injury is defined as an elevated cTn value above the 99th percentile URL and this definition emphasizes on the benefits of hs-cTns determination. ${ }^{71}$ This high-sensitivity determination increases the ability of the biomarker to determine small differences over time and will improve clinical practice.

The main difference between the former cTn assays and hs-cTn assays is the increase in sensitivity, which is only apparent at values near the 99 th percentile (URL). ${ }^{78}$ Different clinical conditions may influence levels, including stable coronary artery disease (CAD), chronic HF, renal dysfunction, sepsis, critically ill patients, acute pulmonary embolism, or pulmonary arterial hypertension.

One of the differences of the recent 4 th definition compared with the previous 3rd definition of MI is related with sex reference levels of troponins for MI diagnosis. Differences between men and women in levels of hs-cTn to the diagnosis of MI and prognostic performance have been observed. ${ }^{79}$ Significantly lower values are observed among women compared with men, and therefore sex-specific 99th percentile URLs are recommended for hs-cT assays. Sex-specific cut-off values have been reported to improve diagnostic and prognostic information in patients with possible acute MI. ${ }^{71,80}$
Humphries et $\mathrm{al}^{81}$ demonstrated different prognosis in women with chest pain in the emergency room; for example, even when females have cardiac chest pain and cTn levels $>$ 99th percentile, they are less likely to be diagnosed with MI, less likely to undergo diagnostic cardiac catheterization within 7 days, and less likely to use evidence-based cardiac medications, but they have the highest 1 -year MACE rate. For that reason, it is important to use specific hs-cTn range (above the 99th percentile URL) for women to avoid underdiagnosis of MI.

Nowadays, there are many different cardiac troponins (cTn) available, cTnT, cTnI, and the high-sensitive hs-cTnT and hs-cTnI. Even though the results from these various testing platform systems may yield similar clinical interpretation for diagnosis, that is, above or below the 99th percentile of the assay with a rise and or fall of cTn, there are considerable differences in the numerical cTn values between assays. This variability may be due to differences in assay calibration, use of different antibodies, differences in assay design, instrument limitations, multiple detection technologies, and differences in the measuring, that is, some assays measure cTnI and others cTnT.

Other important prognosis marker in patients with ACS is renal function. Patients with AMI and severe renal disease 
generally have a poor prognosis. Several registries have shown an increase of bleeding and mortality after ACS and received less aggressive therapy than patients with normal renal function. However, bleeding risk was not increased with the use of third-generation $\mathrm{P}_{2} \mathrm{Y}_{12}$ (ticagrelor and prasugrel) in patients with renal impairment ${ }^{82}$ and these patients are at high risk of mortality and ischemic risk; therefore, it is mandatory to take the most effective therapy.

Other myocardial stress biomarkers such as NT-proBNP have also been analyzed and high values are powerful prognostic markers. Indeed, the combination with cardiac troponin improves risk stratification in NSTEMI; however, the routine use of NT-proBNP in the diagnostic process of ACS in a patient with chest pain is not supported by the current evidence. ${ }^{74}$

Also, there are studies that have analyzed the influence of inflammatory biomarkers in the pathogenesis of CAD due to the proinflammatory mediators involved in atherosclerosis progression. One of these markers is adiponectin. Adiponectin levels are associated not only with CAD presence, but also with CAD extension or severity. Moreover, they can be a good predictor of CAD. Other biomarkers are anticardiolipin antibodies of the immunoglobulin $\mathrm{G}$ isotype ( $\operatorname{IgG} \mathrm{aCL}$ ). These biomarkers were suggested as risk factor for arterial and venous thrombosis. Pastori et $\mathrm{al}^{83}$ analyzed the role of $\operatorname{IgG}$ $\mathrm{aCL}$ in patients with CAD and concluded that patients with elevated IgG aCL levels have a doubled risk of recurrent MACEs and should be suspected in patients with juvenile CAD. However, the use of adiponectin or IgG aCL in daily clinical practice to guide therapy management is limited. ${ }^{84}$

Biomarkers are also included in the risk score assessments of ACS. The GRACE score includes renal function and elevated cardiac troponin levels as biomarkers. The GRACE scores provided superior discrimination as compared to the TIMI NSTEMI score in predicting in-hospital and 6-month mortality in NSTEMI patients, although the GRACE and TIMI STEMI scores performed equally well in STEMI patients. ${ }^{85}$ Indeed, an update of GRACE score (Grace 2.0) was also validated in 32,037 patients from the GRACE registry (14 countries, 94 hospitals) and externally validated in the French registry of Acute ST-elevation and non-ST-elevation MI (FAST-MI). This update had better discrimination, is easier to use, and performed equally well acutely and over the long-term events. ${ }^{86}$ The use of GRACE risk score could help in prognosis of patients after NSTEMI and guide antiplatelet therapy.

\section{Biomarkers in the Prevention of Cardiovascular Disease}

The relationship between classical risk factors to CVDs such as hypertension, diabetes, smoke, or dyslipidemia is well-known. However, some patients present CVD without elevation of traditional risk factors. In this context, the role of biomarkers for primary prevention of CVDs has emerged, trying to assess a $C V$ risk profile of the patients. CRP, apolipoprotein B (apoB), homocysteine, albuminuria, or reactive oxidant species (ROS) have been proposed as biomarkers involved in atherosclerotic process and CV disease progression. Formal clinical guidelines recommendations in - Table 5 .
2016 European Guidelines on Cardiovascular Disease Prevention in Clinical Practice 87

- CV circulating and urinary biomarkers exhibit either no or only limited value when added to CVD risk assessment with the SCORE system. There is evidence of publication bias in the field of novel biomarkers of CV risk, leading to inflated estimates of strength of association and potential added value.

- Not all potentially useful circulatory and urinary biomarkers have undergone state-of-the-art assessment of their added value in CV risk prediction on top of conventional risk factors.

- Biomarkers may be useful in specific subgroups, but this has been addressed in only a limited number of studies.

2019 ACC/AHA Guideline on the Primary Prevention of Cardiovascular Disease ${ }^{88}$

- In adults at borderline risk ( 5 to $<7.5 \% 10$-year CV risk) or intermediate risk ( $\geq 7.5$ to $<20 \% 10$-year ASCVD risk), it is reasonable to use additional risk enhancing factors to guide decisions about preventive interventions (e.g., statin therapy).

- For individuals with intermediate predicted risk ( $\geq 7.5$ to $<20 \%$ ) or for select adults with borderline (5 to $<7.5 \%$ ) predicted risk, coronary artery calcium measurement can be a useful tool in refining risk assessment for preventive interventions (e.g., statin therapy). Coronary artery calcium scoring has superior discrimination and risk reclassification as compared with other subclinical imaging markers or biomarkers.

\section{Comment on Recommendations in the Clinical Guidelines}

Different biomarkers have been proposed to improve CV risk classification especially in patients at intermediate risk with the common clinical factors.

The inflammatory biomarker CRP is one of the most frequently analyzed. CRP binds to low-density lipoprotein and is present in atherosclerotic plaques, so it has been proposed as a causal role in coronary heart disease. The Emerging Risk Factors Collaboration group ${ }^{89}$ performed a meta-analysis with individual record of 160,309 without history of CVD and analyzed the association between CRP and mortality, as well as other adverse outcomes. The authors observed that CRP concentration has continuous association with the risk of coronary heart disease, ischemic stroke, vascular mortality, and death from several cancers and lung disease. However, CRP has a role in very diverse diseases and in some studies with small effect, thus it is difficult to certain that the final data are not simply a result of residual confounding or selection bias due its influence with other risk factors.

Other biomarkers such as apoB and homocysteine have also been analyzed. Wald et $\mathrm{al}^{90}$ performed a meta-analysis to analyze the relationship between serum homocysteine concentration with ischemic heart disease, deep vein thrombosis, and pulmonary embolism. The authors observed a significant association between homocysteine and these three diseases and lowering homocysteine concentrations 
Table 5 Main current clinical guidelines recommendations for biomarkers use in cardiovascular disease prevention

\begin{tabular}{|c|c|c|}
\hline & ESC (86) & AHA/ACC (87) \\
\hline $\begin{array}{l}\text { Cardiovascular } \\
\text { disease }\end{array}$ & $\begin{array}{l}\text { - Routine assessment of circulating or urinary bio- } \\
\text { markers is not recommended for refinement of } \\
\text { CVD risk stratification } \\
\text { Class III, level of evidence B } \\
\text { - The role of metabolomics as risk factors for CVD } \\
\text { and to improve CV risk prediction beyond conven- } \\
\text { tional risk factors should be further assessed }\end{array}$ & $\begin{array}{l}\text { - Assessment of risk-enhancing factors: } \\
\text { Lipids/biomarkers: associated with increased car- } \\
\text { diovascular risk: } \\
\text { - Persistently elevated primary hypertriglyceride- } \\
\text { mia ( } \geq 175 \mathrm{mg} / \mathrm{dL} \text {, nonfasting) } \\
\text { - If measured: } \\
{ }^{* *} \text { Elevated high-sensitivity C-reactive protein } \\
(\geq 2.0 \mathrm{mg} / \mathrm{L}) \\
{ }^{* *} \text { Elevated Lp(a): A relative indication for its } \\
\text { measurement is family history of premature } \\
\text { cardiovascular disease } \\
{ }^{* *} \text { An Lp(a) } \geq 50 \text { mg/dL or } \geq 125 \text { nmol/L consti- } \\
\text { tutes a risk-enhancing factor, especially at } \\
\text { higher levels of Lp(a) } \\
\quad * * \text { Elevated apoB ( } \geq 130 \text { mg/dL): A relative indi- } \\
\text { cation for its measurement would be triglyc- } \\
\text { eride } \geq 200 \text { mg/dL. A level } \geq 130 \text { mg/dL } \\
\text { corresponds to an LDL-C }>160 \text { mg/dL and } \\
\text { constitutes a risk-enhancing factor } \\
\text { **Ankle-branchial index ( }<0.9) \\
\text { Class Ila, level of evidence B } \\
\text { - Coronary artery calcium score risk } \\
\text { In intermediate-risk ( } \geq 7.5 \% \text { to }<20 \% 10 \text {-y risk) } \\
\text { adults or selected borderline-risk ( } 5 \% \text { to }<7.5 \% 10 \text { - } \\
\text { y risk) adults in whom a coronary artery calcium } \\
\text { score is measured for the purpose of making a } \\
\text { treatment decision } \\
\text { - If the coronary artery calcium score is zero, it is } \\
\text { reasonable to withhold statin therapy and reassess } \\
\text { in } 5 \text { to } 10 \text { y, as long as higher-risk conditions are } \\
\text { absent } \\
\text { - If coronary artery calcium score is } 1 \text { to } 99 \text {, it is } \\
\text { reasonable to initiate statin therapy for patients } \\
\geq 55 \text { y of age } \\
\text { - If coronary artery calcium score is } 100 \text { or higher or } \\
\text { in the } 75 \text { th percentile or higher, it is reasonable to } \\
\text { initiate statin therapy } \\
\text { Class Ila, level of evidence B }\end{array}$ \\
\hline
\end{tabular}

Abbreviations: ACC, American College of Cardiology; AHA, American Heart Association; CRP, C-reactive protein; CV, cardiovascular; CVD, cardiovascular disease; ESC, European Society of Cardiology; Hs, high sensitive.

by $3 \mu \mathrm{mol} / \mathrm{L}$ would reduce the risk of ischemic heart disease by $16 \%$. Based on these results, Akintoye et $\mathrm{al}^{91}$ proposed a new biomarker score, the $\mathrm{CHAN}_{2} \mathrm{~T}_{3}$ score, a new biomarker score using five biochemical risk markers: CRP, homocysteine, albuminuria, NT-proBNP, and troponin T. A score of $\geq 2$ was associated with improvement in the c-statistic of the pooled cohort equation for the estimation of $\mathrm{CV}$ risk with a combination of traditional risk factors $(0.748$ vs. 0.734 , $p=0.02$ ). Elevated levels of plasma total homocysteine can result from genetic or nutrient-related disturbances in the transsulfuration or remethylation pathways for homocysteine metabolism. ${ }^{92}$ The enzyme 5,10-methylenetetrahydrofolate reductase (MTHFR) catalyzes the methylation of homocysteine to methionine. Inherited mutations in the gene that make the MTHFR enzyme can lead to an enzyme that is not optimally active and, consequently, may lead to elevated homocysteine levels. ${ }^{93}$ However, the causal mechanism of CVD is not clear or if homocysteine is only a marker of an increased clotting risk. ${ }^{90}$
Ma et $\mathrm{al}^{94}$ identified that MTHFR polymorphism was associated with higher homocysteine levels but not with risk of MI. For these controversial results and some methodological limitations, the assessment of homocysteine in daily clinical practice to predict and stratify the CV risk or routinely treatment of patients with elevated homocysteine is not recommended in the current clinical guidelines.

Lipoprotein (a) [Lp(a)] is composed of apoB-100 covalently bound to apoA. Many studies have concluded that $\operatorname{Lp}(\mathrm{a})$ is associated with the prevalence as well as the severity of CAD and future CV events in the general population. ${ }^{95,96}$ Indeed, $\mathrm{Lp}(\mathrm{a})$ is an independent risk factor for atherosclerotic CVD, with elevated levels estimated to be prevalent in $20 \%$ of the population. Observational and genetic evidence strongly support a causal relationship between high plasma concentrations of $\mathrm{Lp}(\mathrm{a})$ and increased risk of atherosclerotic CVDrelated events, such as MI and stroke, and valvular aortic stenosis. ${ }^{97}$ Zhang et al $^{98}$ showed that elevated $\mathrm{Lp}(\mathrm{a})$ levels were independently associated with the presence and 
severity of CAD in patients with diabetes mellitus. For that reason, American Heart Association guidelines recommend the assessment of risk factors in patients at risk of CVD, with measuring of $\operatorname{Lp}(\mathrm{a})$ levels.

In relation to lipid control, another promising biomarker is represented by proprotein convertase subtilisin/kexin type-9 (PCSK9). The PCSK9 inhibitors showed an important role in cholesterol-lipid lowering in patients with ACS and some studies analyzed the pleiotropic role of these inhibitors. Paciullo et al ${ }^{99}$ reviewed the role of PCSK9 in hemostasis and thrombosis, and observed that in experimental mice, PCSK9 knockout mice develop less venous and arterial thrombosis and show reduced in vivo platelet activation upon arterial injury. In the same line, Pastori et al ${ }^{100}$ analyzed the impact of PCSK9 levels in AF patients and observed that plasma PCSK9 levels are associated with an increase of $\mathrm{CV}$ events as well as positive correlation with urinary thromboxane as a mechanism implicated in platelet activation.
Moreover, the assessment of coronary artery calcium as subclinical imaging marker could improve risk score stratification in patients with intermediate predicted risk. In the MESA (Multi-Ethnic Study of Atherosclerosis) trial, the coronary artery calcium score was strongly associated with 10 -year CV risk across age, sex, and racial/ethnic groups, independent of traditional risk factors. ${ }^{101}$ Indeed, a coronary artery calcium score of $<0$ identifies individuals at lower risk of $C V$ events and death over a $\geq 10$-year period. ${ }^{101-103}$ Thus, the absence of coronary artery calcium could reclassify a patient downward into a lower risk group in which preventive interventions (e.g., statins) could be postponed. ${ }^{104}$

A new perspective in $\mathrm{CV}$ risk assessment involves the role of oxidative stress biomarkers. Different studies about biomarkers related with oxidative stress and its influence in CVD are ongoing. A panel of more than 70 biomarkers of oxidative stress, especially related to lipid oxidation and peroxidation, has been developed but there is a lack of
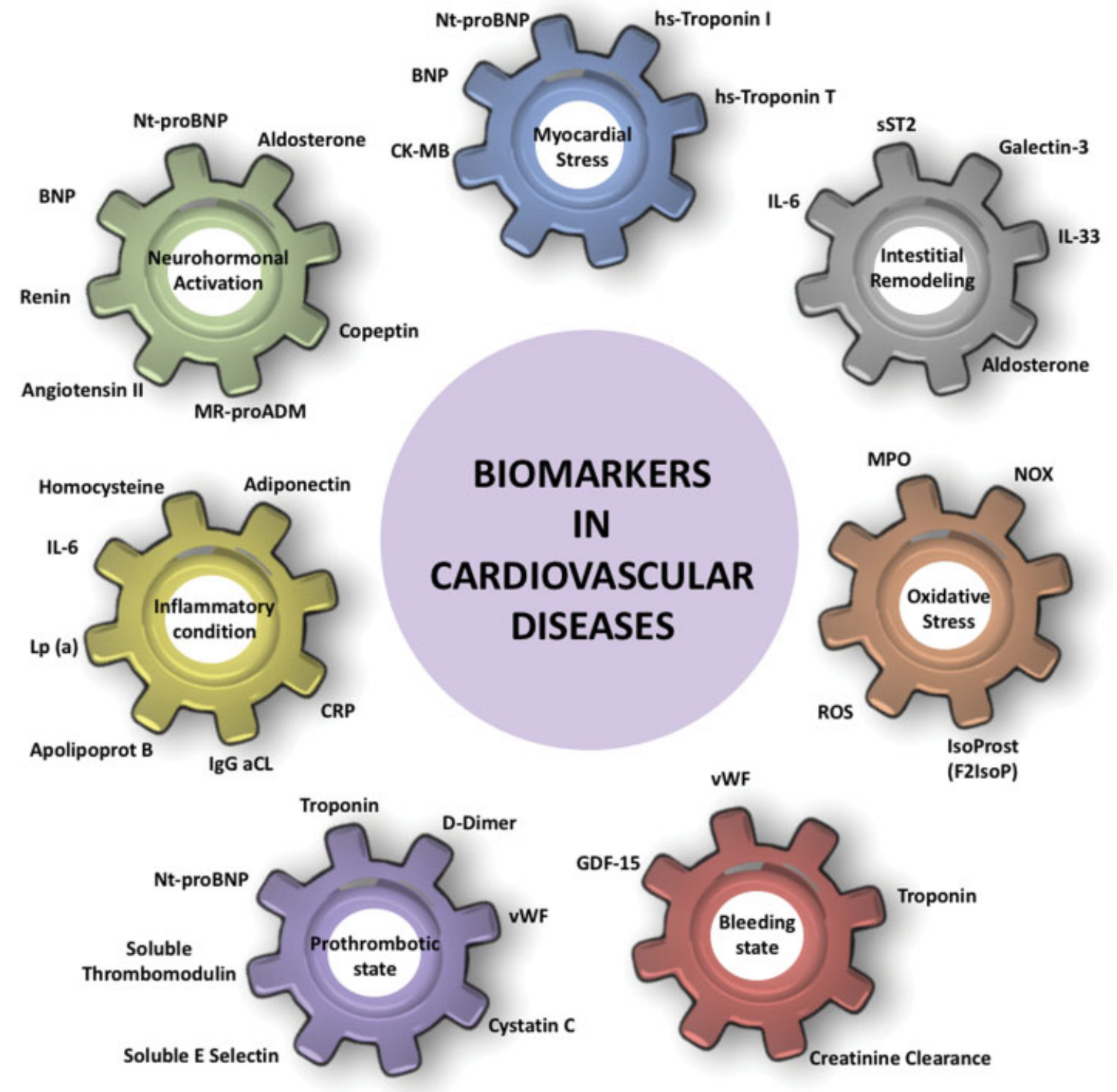

Fig. 1 Different biomarkers involved in cardiovascular diseases. CK-MB, creatine kinase myocardial isoenzyme; CRP, C-reactive protein; GDF-15, growth differentiation factor-15; LP(a), lipoprotein a; MPO, myeloperoxidase; MRproADM, mid-regional pro-adrenomedullin; NOX:Gal-3, galectin 3; NT-proBNP, N-terminal pro-B type natriuretic peptide; ROS, reactive oxidant species; sST2, soluble suppression of tumorigenicity 2. 
validation about the role of biomarkers levels and CV risk profile of patients. ${ }^{105}$ One of the biomarkers are focused in ROS-generating enzymes, NADPH oxidase, and MPO. These enzymes have been suggested to be implicated in the atherosclerosis process and plaque instability. ${ }^{106}$ Pastori et al ${ }^{107}$ performing a review of recent reports focus on NADPH oxidase and MPO measurement and observed disappointing results in interventional studies with antioxidants in primary and secondary prevention of $\mathrm{CV}$ diseases, but the use these biomarkers in the assessment of atherosclerotic risk should be promising.

\section{Conclusion}

Ultimately, a balance is needed between simplicity and practicality for clinical decision-making. Most biomarkers (whether blood, urine, or imaging-based) will improve on clinical risk stratification, but awaiting biomarker results may lead to delays in the initiation of therapy, for example, anticoagulation for stroke prevention in AF. Many biomarkers are nonspecific, being predictive of many $\mathrm{CV}$ and non-CV outcomes, so would be better as "rule-out" rather than "rulein" assessments (-Fig. 1). Derivation of some biomarkers have also been made in highly selected clinical trial cohorts, where measurement is made at baseline but outcomes determined many years later; given the dynamic nature of risk in the "real world" where patients get older and develop incident risk factors, this may give a false impression of the risk profile. Finally, some laboratory biomarkers have a diurnal variation and inter-/intravariability (and lower limits of detection) in assays, which may be expensive, are added considerations.

Indeed, many clinical guidelines recommendations about biomarkers' use in clinical practice reflect the consensus expert opinion, with limited and weak evidence. In this field, the recommendations of clinical guidelines should be analyzed with caution, using consistent, real-world, and all external validation data available to maximize the generalization and uniformity of the recommendations. The final objective should be to help clinicians in decision-making process in daily clinical practice, assessing all the clinical and blood markers to offer the best therapy to patients with CVD. A balance between simplicity and practical application, versus modest improvements in prediction (at least statistically), is needed.

\section{Funding}

This work was supported by Instituto de Salud Carlos III (ISCIII), Fondo Europeo de Desarrollo Regional (FEDER) (Research grant: PI17/01375), and Centro de Investigación Biomédica en Red - Enfermedades Cardiovasculares (CIBER-CV, group reference CB16/11/00385).

\section{Conflict of Interest}

F.M. has received fees for educational purposes for Boehringer-Ingelheim and Daiichi Sankyo and as advisory board of Boehringer-Ingelheim, Bayer, Pfizer, Daiichi Sankyo, and Atrial Fibrillation Network (AFNET). V.R. has received fees for educational purposes for Boehringer-Ingelheim and Pfizer, and investigator fees for Boehringer-Ingelheim. G. Y.L. has served as a consultant for Bayer/Janssen, BMS/Pfizer, Biotronik, Medtronic, Boehringer Ingelheim, Novartis, Verseon, and Daiichi-Sankyo and as a speaker for Bayer, BMS/Pfizer, Medtronic, Boehringer Ingelheim, and Daiichi-Sankyo. No fees were directly received personally. The authors have no other funding, financial relations, or conflicts of interest to disclose related to this work.

\section{References}

1 Goodsaid F, Frueh F. Biomarker qualification pilot process at the US Food and Drug Administration. AAPS J 2007;9(01): E105-E108

2 Rodríguez-Serrano AI, Esteve-Pastor MA, Hernández-Romero D, Valdés M, Roldán V, Marín F. New role of biomarkers in atrial fibrillation. In: Patel VB, Preedy VR, eds. Biomarkers in Cardiovascular Disease. Dordrecht: Springer Netherlands; 2016:507-539

3 Wang J, Tan G-J, Han L-N, Bai Y-Y, He M, Liu H-B. Novel biomarkers for cardiovascular risk prediction. JGeriatr Cardiol 2017;14(02):135-150

4 Chow SL, Maisel AS, Anand I, et al; American Heart Association Clinical Pharmacology Committee of the Council on Clinical Cardiology; Council on Basic Cardiovascular Sciences; Council on Cardiovascular Disease in the Young; Council on Cardiovascular and Stroke Nursing; Council on Cardiopulmonary, Critical Care, Perioperative and Resuscitation; Council on Epidemiology and Prevention; Council on Functional Genomics and Translational Biology; and Council on Quality of Care and Outcomes Research. Role of biomarkers for the prevention, assessment, and management of heart failure: a scientific statement from the American Heart Association. Circulation 2017;135(22):e1054-e1091

5 Ponikowski P, Voors AA, Anker SD, et al; ESC Scientific Document Group. 2016 ESC Guidelines for the diagnosis and treatment of acute and chronic heart failure: the Task Force for the diagnosis and treatment of acute and chronic heart failure of the European Society of Cardiology (ESC). Developed with the special contribution of the Heart Failure Association (HFA) of the ESC. Eur Heart J 2016;37(27):2129-2200

6 Daniels LB, Maisel AS. Natriuretic peptides. JAm Coll Cardiol 2007;50(25):2357-2368

7 Boomsma F, van den Meiracker AH. Plasma A- and B-type natriuretic peptides: physiology, methodology and clinical use. Cardiovasc Res 2001;51(03):442-449

8 Daniels LB, Clopton P, deFilippi CR, et al. Serial measurement of $\mathrm{N}$-terminal pro-B-type natriuretic peptide and cardiac troponin $\mathrm{T}$ for cardiovascular disease risk assessment in the Multi-Ethnic Study of Atherosclerosis (MESA). Am Heart J 2015;170(06): 1170-1183

9 Myhre PL, Vaduganathan M, Claggett B, et al. B-type natriuretic peptide during treatment with sacubitril/valsartan: the PARADIGM-HF trial. J Am Coll Cardiol 2019;73(11):1264-1272

10 Berger R, Moertl D, Peter S, et al. N-terminal pro-B-type natriuretic peptide-guided, intensive patient management in addition to multidisciplinary care in chronic heart failure a 3-arm, prospective, randomized pilot study. J Am Coll Cardiol 2010;55 (07):645-653

11 Fonarow GC, Peacock WF, Horwich TB, et al; ADHERE Scientific Advisory Committee and Investigators. Usefulness of B-type natriuretic peptide and cardiac troponin levels to predict in-hospital mortality from ADHERE. Am J Cardiol 2008;101(02):231-237

12 Kociol RD, Horton JR, Fonarow GC, et al. Admission, discharge, or change in B-type natriuretic peptide and long-term outcomes: data from Organized Program to Initiate Lifesaving Treatment in Hospitalized Patients with Heart Failure (OPTIMIZE-HF) linked to Medicare claims. Circ Heart Fail 2011;4(05):628-636 
13 Brouwers FP, van Gilst WH, Damman K, et al. Clinical risk stratification optimizes value of biomarkers to predict newonset heart failure in a community-based cohort. Circ Heart Fail 2014;7(05):723-731

14 Roberts E, Ludman AJ, Dworzynski K, et al; NICE Guideline Development Group for Acute Heart Failure. The diagnostic accuracy of the natriuretic peptides in heart failure: systematic review and diagnostic meta-analysis in the acute care setting. BMJ 2015;350:h910

15 Maisel A, Mueller C, Adams K Jr, et al. State of the art: using natriuretic peptide levels in clinical practice. Eur J Heart Fail 2008;10(09):824-839

16 Virani SA, Dent S, Brezden-Masley C, et al. Canadian Cardiovascular Society guidelines for evaluation and management of cardiovascular complications of cancer therapy. Can J Cardiol 2016;32(07):831-841

17 Filippatos G, Farmakis D, Parissis J. Renal dysfunction and heart failure: things are seldom what they seem. Eur Heart J 2014;35 (07):416-418

18 Mascarenhas J, Laszczynska O, Severo M, et al. Prognostic effect of renal function in ambulatory patients with heart failure and reduced ejection fraction: the kidney is a marker of cardiac function. Can J Cardiol 2018;34(10):1325-1332

19 Camm AJ, Lip GYH, De Caterina R, et al; ESC Committee for Practice Guidelines (CPG). 2012 focused update of the ESC Guidelines for the management of atrial fibrillation: an update of the 2010 ESC Guidelines for the management of atrial fibrillation. Developed with the special contribution of the European Heart Rhythm Association. Eur Heart J 2012;33(21):2719-2747

20 Ky B, French B, McCloskey K, et al. High-sensitivity ST2 for prediction of adverse outcomes in chronic heart failure. Circ Heart Fail 2011;4(02):180-187

21 Lok DJA, Van Der Meer P, de la Porte PWB-A, et al. Prognostic value of galectin-3, a novel marker of fibrosis, in patients with chronic heart failure: data from the DEAL-HF study. Clin Res Cardiol 2010;99(05):323-328

22 Daniels LB, Clopton P, Laughlin GA, Maisel AS, Barrett-Connor E. Galectin-3 is independently associated with cardiovascular mortality in community-dwelling older adults without known cardiovascular disease: the Rancho Bernardo Study. Am Heart J 2014;167 (05):674-682

23 Hart RG, Pearce LA, Aguilar MI. Meta-analysis: antithrombotic therapy to prevent stroke in patients who have nonvalvular atrial fibrillation. Ann Intern Med 2007;146(12):857-867

24 Hijazi Z, Oldgren J, Siegbahn A, Granger CB, Wallentin L. Biomarkers in atrial fibrillation: a clinical review. Eur Heart J 2013; 34(20):1475-1480

25 VílchezJA, Orenes-Piñero E, Hernández-Romero D, Valdés M, Marín F. Biomarkers of necrosis and myocardial remodeling. In: Preedy VR, Patel VB, eds. General Methods in Biomarker Research and Their Applications. Dordrecht: Springer Netherlands; 2014:1-24

26 Lip GYH, Banerjee A, Boriani G, et al. Antithrombotic therapy for atrial fibrillation: CHEST Guideline and Expert Panel Report. Chest 2018;154(05):1121-1201

27 Kirchhof P, Benussi S, Kotecha D, et al; ESC Scientific Document Group. 2016 ESC Guidelines for the management of atrial fibrillation developed in collaboration with EACTS. Eur Heart J 2016;37(38):2893-2962

28 January CT, Wann LS, Alpert JS, et al. American College of Cardiology/American Heart Association Task Force on Practice Guidelines. 2014 AHA/ACC/HRS Guideline for the Management of Patients With Atrial Fibrillation: Executive Summary: a report of the American College of Cardiology/American Heart Association Task Force on Practice Guidelines and the Heart Rhythm Society. J Am Coll Cardiol 2014;64(21):2246-2280

29 Aviles RJ, Martin DO, Apperson-Hansen C, et al. Inflammation as a risk factor for atrial fibrillation. Circulation 2003;108(24): 3006-3010
30 Patton KK, Ellinor PT, Heckbert SR, et al. N-terminal pro-B-type natriuretic peptide is a major predictor of the development of atrial fibrillation: the Cardiovascular Health Study. Circulation 2009;120(18):1768-1774

31 Chua W, Purmah Y, Cardoso VR, et al. Data-driven discovery and validation of circulating blood-based biomarkers associated with prevalent atrial fibrillation. Eur Heart J 2019;40(16): 1268-1276

32 Lip G, Freedman B, De Caterina R, Potpara TS. Stroke prevention in atrial fibrillation: past, present and future. Comparing the guidelines and practical decision-making. Thromb Haemost 2017;117(07):1230-1239

33 January CT, Wann LS, Calkins H, et al. 2019 AHA/ACC/HRS Focused Update of the 2014 AHA/ACC/HRS Guideline for the Management of Patients With Atrial Fibrillation: a report of the American College of Cardiology/American Heart Association Task Force on Clinical Practice Guidelines and the Heart Rhythm Society. J Am Coll Cardiol 2019;74(01):104-132

34 Borre ED, Goode A, Raitz G, et al. Predicting thromboembolic and bleeding event risk in patients with non-valvular atrial fibrillation: a systematic review. Thromb Haemost 2018;118(12): 2171-2187

35 Proietti M, Mujovic N, Potpara TS. Optimizing stroke and bleeding risk assessment in patients with atrial fibrillation: a balance of evidence, practicality and precision. Thromb Haemost 2018; 118(12):2014-2017

36 Lip GYH, Lane D, Van Walraven C, Hart RG. Additive role of plasma von Willebrand factor levels to clinical factors for risk stratification of patients with atrial fibrillation. Stroke 2006;37 (09):2294-2300

37 García-Fernández A, Roldán V, Rivera-Caravaca JM, et al. Does von Willebrand factor improve the predictive ability of current risk stratification scores in patients with atrial fibrillation? Sci Rep 2017; 7:41565

38 Van Wagoner DR. Oxidative stress and inflammation in atrial fibrillation: role in pathogenesis and potential as a therapeutic target. J Cardiovasc Pharmacol 2008;52(04):306-313

39 Violi F, Pignatelli P, Pignata C, et al. Reduced atherosclerotic burden in subjects with genetically determined low oxidative stress. Arterioscler Thromb Vasc Biol 2013;33(02):406-412

40 Pignatelli P, Pastori D, Carnevale R, et al. Serum NOX2 and urinary isoprostanes predict vascular events in patients with atrial fibrillation. Thromb Haemost 2015;113(03):617-624

41 van den Bos EJ, Constantinescu AA, van Domburg RT, Akin S, Jordaens LJ, Kofflard MJM. Minor elevations in troponin I are associated with mortality and adverse cardiac events in patients with atrial fibrillation. Eur Heart J 2011;32(05):611-617

42 Roldán V, Marín F, Díaz J, et al. High sensitivity cardiac troponin T and interleukin- 6 predict adverse cardiovascular events and mortality in anticoagulated patients with atrial fibrillation. J Thromb Haemost 2012;10(08):1500-1507

43 Hijazi Z, Oldgren J, Andersson U, et al. Cardiac biomarkers are associated with an increased risk of stroke and death in patients with atrial fibrillation: a Randomized Evaluation of Long-term Anticoagulation Therapy (RE-LY) substudy. Circulation 2012;125 (13):1605-1616

44 Hijazi Z, Wallentin L, Siegbahn A, et al; ARISTOTLE Investigators. High-sensitivity troponin $T$ and risk stratification in patients with atrial fibrillation during treatment with apixaban or warfarin. J Am Coll Cardiol 2014;63(01):52-61

45 Roldán V, Vílchez JA, Manzano-Fernández S, et al. Usefulness of $\mathrm{N}$-terminal pro-B-type natriuretic Peptide levels for stroke risk prediction in anticoagulated patients with atrial fibrillation. Stroke 2014;45(03):696-701

46 Hijazi Z, Lindbäck J, Alexander JH, et al; ARISTOTLE and STABILITY Investigators. The ABC (age, biomarkers, clinical history) stroke risk score: a biomarker-based risk score for predicting stroke in atrial fibrillation. Eur Heart J 2016;37(20):1582-1590 
47 Rivera-Caravaca JM, Roldán V, Esteve-Pastor MA, et al. Long-term stroke risk prediction in patients with atrial fibrillation: comparison of the $\mathrm{ABC}$-Stroke and $\mathrm{CHA}_{2} \mathrm{DS}_{2}$-VASc scores. J Am Heart Assoc 2017;6(07):e006490

48 Esteve-Pastor MA, Rivera-Caravaca JM, Roldan V, et al. Long-term bleeding risk prediction in 'real world' patients with atrial fibrillation: comparison of the HAS-BLED and ABC-Bleeding risk scores. The Murcia Atrial Fibrillation Project. Thromb Haemost 2017;117 (10):1848-1858

49 Lip GYH. Assessing bleeding risk with the HAS-BLED score: balancing simplicity, practicality, and predictive value in bleeding-risk assessment. Clin Cardiol 2015;38(09):562-564

50 Lip GYH, Frison L, Halperin JL, Lane DA. Comparative validation of a novel risk score for predicting bleeding risk in anticoagulated patients with atrial fibrillation: the HAS-BLED (Hypertension, Abnormal Renal/Liver Function, Stroke, Bleeding History or Predisposition, Labile INR, Elderly, Drugs/Alcohol Concomitantly) score. JAm Coll Cardiol 2011;57(02):173-180

51 Hijazi Z, Oldgren J, Lindbäck J, et al; ARISTOTLE and RE-LY Investigators. The novel biomarker-based ABC (age, biomarkers, clinical history)-bleeding risk score for patients with atrial fibrillation: a derivation and validation study. Lancet 2016;387 (10035):2302-2311

52 Apostolakis S, Lane DA, Guo Y, Buller H, Lip GYH. Performance of the HEMORR(2)HAGES, ATRIA, and HAS-BLED bleeding riskprediction scores in patients with atrial fibrillation undergoing anticoagulation: the AMADEUS (evaluating the use of SR34006 compared to warfarin or acenocoumarol in patients with atrial fibrillation) study. J Am Coll Cardiol 2012;60(09):861-867

53 Zulkifly H, Lip GYH, Lane DA. Bleeding risk scores in atrial fibrillation and venous thromboembolism. Am J Cardiol 2017; 120(07):1139-1145

54 Roldán V, Marín F, Muiña B, et al. Plasma von Willebrand factor levels are an independent risk factor for adverse events including mortality and major bleeding in anticoagulated atrial fibrillation patients. J Am Coll Cardiol 2011;57(25):2496-2504

55 Hijazi Z, Wallentin L, Siegbahn A, et al. N-terminal pro-B-type natriuretic peptide for risk assessment in patients with atrial fibrillation: insights from the ARISTOTLE Trial (Apixaban for the Prevention of Stroke in Subjects With Atrial Fibrillation). JAm Coll Cardiol 2013;61(22):2274-2284

56 Wallentin L, Hijazi Z, Andersson U, et al; ARISTOTLE Investigators. Growth differentiation factor 15, a marker of oxidative stress and inflammation, for risk assessment in patients with atrial fibrillation: insights from the Apixaban for Reduction in Stroke and Other Thromboembolic Events in Atrial Fibrillation (ARISTOTLE) trial. Circulation 2014;130(21):1847-1858

57 Hijazi Z, Oldgren J, Andersson U, et al. Growth-differentiation factor 15 and risk of major bleeding in atrial fibrillation: insights from the Randomized Evaluation of Long-Term Anticoagulation Therapy (RE-LY) trial. Am Heart J 2017;190:94-103

58 Sharma A, Stevens SR, Lucas J, et al. Utility of growth differentiation factor-15, a marker of oxidative stress and inflammation, in chronic heart failure: insights from the HF-ACTION study. JACC Heart Fail 2017;5(10):724-734

59 Ban N, Siegfried CJ, Lin JB, et al. GDF15 is elevated in mice following retinal ganglion cell death and in glaucoma patients. JCI Insight 2017;2(09):91455

60 Connolly SJ, Ezekowitz MD, Yusuf S, et al; RE-LY Steering Committee and Investigators. Dabigatran versus warfarin in patients with atrial fibrillation. N Engl J Med 2009;361(12): 1139-1151

61 Rivera-Caravaca JM, Esteve-Pastor MA, Marín F, et al. A propensity score matched comparison of clinical outcomes in atrial fibrillation patients taking vitamin K antagonists: comparing the "real-world" vs clinical trials. Mayo Clin Proc 2018;93(08):1065-1073

62 Yoon M, Yang P-S, Jang E, et al. Dynamic changes of CHA2DS2VASc score and the risk of ischaemic stroke in Asian patients with atrial fibrillation: a nationwide cohort study. Thromb Haemost 2018;118(07):1296-1304

63 Williams JA, Lalonde R, Koup JR, Christ DD. Predictive Approaches in Drug Discovery and Development: Biomarkers and In Vitro/In Vivo Correlations. Hoboken, NJ: John Wiley \& Sons; 2012. ISBN 978-0-470-17083-0

64 January CT, Wann LS, Calkins H, et al. 2019 AHA/ACC/HRS focused update of the 2014 AHA/ACC/HRS guideline for the management of patients with atrial fibrillation: a report of the American College of Cardiology/American Heart Association Task Force on Clinical Practice Guidelines and the Heart Rhythm Society in Collaboration With the Society of Thoracic Surgeons. Circulation 2019;140(02):e125-e151

65 Esteve-Pastor MA, Rivera-Caravaca JM, Roldán-Rabadán I, et al; FANTASIIA Study Investigators. Relation of renal dysfunction to quality of anti coagulation control inpatients with atrialfibrillation: the FANTASIIA Registry. Thromb Haemost 2018;118(02): 279-287

66 Roldán V, Marín F, Manzano-Fernandez S, Fernández H, Gallego P, Valdés $M$, et al. Does chronic kidney disease improve the predictive value of the CHADS2 and CHA2DS2-VASc stroke stratification risk scores for atrial fibrillation? Thromb Haemost 2013;109(05):956-960

67 Bonde AN, Lip GYH, Kamper A-L, et al. Net clinical benefit of antithrombotic therapy in patients with atrial fibrillation and chronic kidney disease: a nationwide observational cohort study. JAm Coll Cardiol 2014;64(23):2471-2482

68 Nielsen PB, Lane DA, Rasmussen LH, Lip GYH, Larsen TB. Renal function and non-vitamin $\mathrm{K}$ oral anticoagulants in comparison with warfarin on safety and efficacy outcomes in atrial fibrillation patients: a systemic review and meta-regression analysis. Clin Res Cardiol 2015;104(05):418-429

69 Roldán V, Marín F, Fernández H, et al. Renal impairment in a "real-life" cohort of anticoagulated patients with atrial fibrillation (implications for thromboembolism and bleeding). Am J Cardiol 2013;111(08):1159-1164

70 Fauchier L, Bisson A, Clementy N, et al. Changes in glomerular filtration rate and outcomes in patients with atrial fibrillation. Am Heart J 2018;198:39-45

71 Thygesen K, Alpert JS, Jaffe AS, Chaitman BR, Bax JJ, Morrow DA, et al. Fourth universal definition of myocardial infarction (2018). Eur Heart J 2019;40(03):237-269

72 Ibanez B, James S, Agewall S, et al. ESC Guidelines for the management of acute myocardial infarction in patients presenting with ST-segment elevation: the Task Force for the management of acute myocardial infarction in patients presenting with ST-segment elevation of the European Society of Cardiology (ESC). Eur Heart J 2018;39(02):119-177

73 Amsterdam EA, Wenger NK, Brindis RG, et al. 2014 AHA/ACC Guideline for the Management of Patients with Non-ST-Elevation Acute Coronary Syndromes: a report of the American College of Cardiology/American Heart Association Task Force on Practice Guidelines. JAm Coll Cardiol 2014;64(24):e139-e228

74 Roffi M, Patrono C, Collet JP, et al. ESC Scientific Document Group. ESC Guidelines for the management of acute coronary syndromes in patients presenting without persistent ST-segment elevation: Task Force for the Management of Acute Coronary Syndromes in Patients Presenting without Persistent ST-Segment Elevation of the European Society of Cardiology (ESC). Eur Heart J 2016;37(03):267-315

75 Mueller C. Biomarkers and acute coronary syndromes: an update. Eur Heart J 2014;35(09):552-556

76 Reichlin T, Hochholzer W, Bassetti S, et al. Early diagnosis of myocardial infarction with sensitive cardiac troponin assays. N Engl J Med 2009;361(09):858-867

77 Twerenbold R, Neumann JT, Sörensen NA, et al. Prospective validation of the $0 / 1$-h algorithm for early diagnosis of myocardial infarction. J Am Coll Cardiol 2018;72(06):620-632 
78 Thygesen K, Mair J, Giannitsis E, et al; Study Group on Biomarkers in Cardiology of ESC Working Group on Acute Cardiac Care. How to use high-sensitivity cardiac troponins in acute cardiac care. Eur Heart J 2012;33(18):2252-2257

79 Balmelli C, Meune C, Twerenbold R, et al. Comparison of the performances of cardiac troponins, including sensitive assays, and copeptin in the diagnostic of acute myocardial infarction and long-term prognosis between women and men. Am Heart J 2013;166(01):30-37

80 Kimenai DM, Henry RMA, van der Kallen $\mathrm{CJH}$, et al. Direct comparison of clinical decision limits for cardiac troponin $\mathrm{T}$ and I. Heart 2016;102(08):610-616

81 Humphries KH, Lee MK, Izadnegahdar M, et al. Sex differences in diagnoses, treatment, and outcomes for emergency department patients with chest pain and elevated cardiac troponin. Acad Emerg Med 2018;25(04):413-424

82 Tello-Montoliu A, Ruiz-Nodar JM, Esteve-Pastor MA, et al. Chronic kidney disease and third-generation P2Y12 inhibitors use in patients with acute coronary syndrome: impact on the prognosis at 1 year. JClin Pharmacol 2019;59(02):295-302

83 Pastori D, Bucci T, Triggiani M, et al. Immunoglobulin G (IgG) anticardiolipin antibodies and recurrent cardiovascular events. A systematic review and Bayesian meta-regression analysis. Autoimmun Rev 2019;18(05):519-525

84 Persson J, Folkersen L, Ekstrand J, et al. High plasma adiponectin concentration is associated with all-cause mortality in patients with carotid atherosclerosis. Atherosclerosis 2012;225(02):491-496

85 Aragam KG, Tamhane UU, Kline-Rogers E, et al. Does simplicity compromise accuracy in ACS risk prediction? A retrospective analysis of the TIMI and GRACE risk scores. PLoS One 2009;4(11): e7947

86 Fox KAA, Fitzgerald G, Puymirat E, et al. Should patients with acute coronary disease be stratified for management according to their risk? Derivation, external validation and outcomes using the updated GRACE risk score. BMJ Open 2014;4(02):e004425

87 Piepoli MF, Hoes AW, Agewall S, et al; ESC Scientific Document Group. 2016 European Guidelines on cardiovascular disease prevention in clinical practice: the Sixth Joint Task Force of the European Society of Cardiology and Other Societies on Cardiovascular Disease Prevention in Clinical Practice (constituted by representatives of 10 societies and by invited experts) Developed with the special contribution of the European Association for Cardiovascular Prevention \& Rehabilitation (EACPR). Eur Heart J 2016;37(29):2315-2381

88 Arnett DK, Blumenthal RS, Albert MA, et al. 2019 ACC/AHA guideline on the primary prevention of cardiovascular disease: Executive summary: a report of the American College of Cardiology/American Heart Association Task Force on Clinical Practice Guidelines. Circulation 2019;pii:S0735-1097(19)33876-8

89 Kaptoge S, Di Angelantonio E, Lowe G, et al; Emerging Risk Factors Collaboration. C-reactive protein concentration and risk of coronary heart disease, stroke, and mortality: an individual participant meta-analysis. Lancet 2010;375(9709):132-140

90 Wald DS, Law M, Morris JK. Homocysteine and cardiovascular disease: evidence on causality from a meta-analysis. BMJ 2002; 325(7374): 1202

91 Akintoye E, Briasoulis A, Afonso L. Biochemical risk markers and 10 -year incidence of atherosclerotic cardiovascular disease: independent predictors, improvement in pooled cohort equation, and risk reclassification. Am Heart J 2017;193:95-103

92 Kang SS, Passen EL, Ruggie N, Wong PW, Sora H. Thermolabile defect of methylenetetrahydrofolate reductase in coronary artery disease. Circulation 1993;88(4 Pt 1):1463-1469

93 Moll S, Varga EA. Homocysteine and MTHFR mutations. Circulation 2015;132(01):e6-e9

$94 \mathrm{Ma}$ J, Stampfer MJ, Hennekens $\mathrm{CH}$, et al. Methylenetetrahydrofolate reductase polymorphism, plasma folate, homocysteine, and risk of myocardial infarction in US physicians. Circulation 1996;94(10):2410-2416

95 Kamstrup PR, Tybjærg-Hansen A, Nordestgaard BG. Extreme lipoprotein(a) levels and improved cardiovascular risk prediction.J Am Coll Cardiol 2013;61(11):1146-1156

96 Erqou S, Kaptoge S, Perry PL, et al; Emerging Risk Factors Collaboration. Lipoprotein(a) concentration and the risk of coronary heart disease, stroke, and nonvascular mortality. JAMA 2009;302(04):412-423

97 Wilson DP, Jacobson TA, Jones PH, et al. Use of lipoprotein(a) in clinical practice: A biomarker whose time has come. A scientific statement from the National Lipid Association. J Clin Lipidol 2019;13(03):374-392

98 Zhang H-W, Zhao X, Guo Y-L, et al. Elevated lipoprotein (a) levels are associated with the presence and severity of coronary artery disease in patients with type 2 diabetes mellitus. Nutr Metab Cardiovasc Dis 2018;28(10):980-986

99 Paciullo F, Momi S, Gresele P. PCSK9 in haemostasis and thrombosis: possible pleiotropic effects of PCSK9 inhibitors in cardiovascular prevention. Thromb Haemost 2019;119(03): 359-367

100 Pastori D, Nocella C, Farcomeni A, et al; ATHERO-AF Study Group. Relationship of PCSK9 and urinary thromboxane excretion to cardiovascular events in patients with atrial fibrillation. JAm Coll Cardiol 2017;70(12):1455-1462

101 Budoff MJ, Young R, Burke G, et al. Ten-year association of coronary artery calcium with atherosclerotic cardiovascular disease (ASCVD) events: the multi-ethnic study of atherosclerosis (MESA). Eur Heart J 2018;39(25):2401-2408

102 DeFilippis AP, Young R, Carrubba CJ, et al. An analysis of calibration and discrimination among multiple cardiovascular risk scores in a modern multiethnic cohort. Ann Intern Med 2015;162(04):266-275

103 Valenti V, Ó Hartaigh B, Heo R, et al. A 15-year warranty period for asymptomatic individuals without coronary artery calcium: a prospective follow-up of 9,715 individuals. JACC Cardiovasc Imaging 2015;8(08):900-909

104 Blaha MJ, Cainzos-Achirica M, Greenland P, et al. Role of coronary artery calcium score of zero and other negative risk markers for cardiovascular disease: the Multi-Ethnic Study of Atherosclerosis (MESA). Circulation 2016;133(09):849-858

105 Cipak Gasparovic A, Zarkovic N, Zarkovic K, et al. Biomarkers of oxidative and nitro-oxidative stress: conventional and novel approaches. Br J Pharmacol 2017;174(12):1771-1783

106 Frijhoff J, Winyard PG, Zarkovic N, et al. Clinical relevance of biomarkers of oxidative stress. Antioxid Redox Signal 2015;23(14): $1144-1170$

107 Pastori D, Carnevale R, Pignatelli P. Is there a clinical role for oxidative stress biomarkers in atherosclerotic diseases? Intern Emerg Med 2014;9(02):123-131 